\title{
Pole-InduCed Higgs Inflation With HyPerbolic KähleR GEOMETRIES
}

\author{
C. Pallis \\ Laboratory of Physics, Faculty of Engineering, \\ Aristotle University of Thessaloniki, \\ GR-541 24 Thessaloniki, GREECE \\ e-mail address: kpallis@gen.auth.gr
}

\begin{abstract}
We present novel realizations of Higgs inflation within Supergravity which are largely tied to the existence of a pole of order two in the kinetic term of the inflaton field. This pole arises due to the selected Kähler potentials which parameterize the $(S U(1,1) / U(1))^{2}$ or $S U(2,1) /(S U(2) \times U(1))$ manifolds with scalar curvatures $\mathcal{R}_{(11)^{2}}=-4 / N$ or $\mathcal{R}_{21}=$ $-3 / N$ respectively. The associated superpotential includes, in addition to the Higgs superfields, a stabilizer superfield, respects the gauge and an $R$ symmetries and contains the first allowed nonrenormalizable term. If the coefficient of this term is almost equal to that of the others within about $10^{-5}$ and $N=1$, the inflationary observables can be done compatible with the present data and the scale $M$ of gauge-symmetry breaking may assume its value within MSSM. Increasing $M$ beyond this value, though, inflation may be attained with less tuning. Modifications to the Kähler potentials associated with the manifolds above allow for inflation, realized with just renormalizable superpotential terms, which results to higher tensor-to-scalar ratios as $N$ approaches its maximum at $N \simeq 40$.

Keywords: Cosmology of Theories Beyond the Standard Model, Supergravity Models;

PACS codes: 98.80.Cq, 11.30.Qc, 12.60.Jv, 04.65.+e
\end{abstract}

Published in J. Cosmol. Astropart. Phys. 05, 043 (2021) 


\section{CONTENTS}

1 INTRODUCTION

2 SUPERgRaVity Framework 4

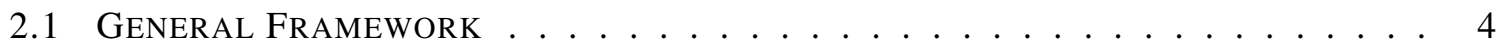

2.2 CANONICALly NoRMALIZED FILEDS . . . . . . . . . . . . . . 5

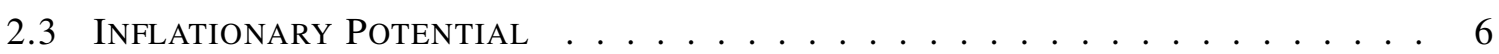

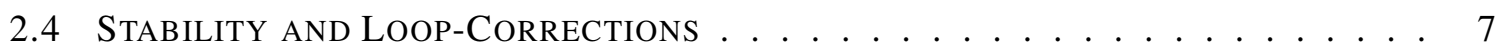

3 InFLation ANALYSIS 9

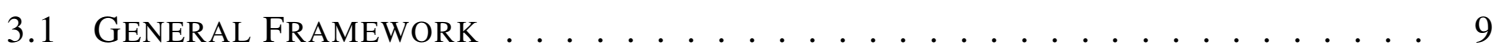

3.2 ANALYTIC RESUlts . . . . . . . . . . . . . . . . . . . . . . . . . . . . . . . . 11

3.3 NUMERICAL RESUlts . . . . . . . . . . . . . . . . . . . . . . . 13

4 Conclusions

Appendix A: Mathematical Supplement

REFERENCES

\section{INTRODUCTION}

The plethora of recent [1-4] and forthcoming [5-7] data on the cosmic microwave background (radiation) (CMB) are stimulating a new wave of inflationary model-building [8, 9]. One of the central task for the successful embedding of an inflationary model within Particle Physics is the identification of the inflaton - i.e., the scalar field causing inflation - with one of the fields already present in the fundamental theory. According to an economical and intriguing set-up, the inflaton could play, at the end of its inflationary evolution, the role of a Higgs field [10-20] leading to a spontaneous breaking of a gauge group. The relevant models may be called Higgs inflation (HI) for short. Let us clarify here that with this term we do not restrict our attention only to the inflationary models [11,14] which exclusively utilize the electroweak Higgs field as higgsflaton [12].

Working in the context of Supergravity (SUGRA) we find it technically convenient to exemplify HI taking as reference theory an "elementary" supersymmetric (SUSY) Grand Unified Theory (GUT) based on the gauge group $G_{B-L}=G_{\mathrm{SM}} \times U(1)_{B-L}$ - where $G_{\mathrm{SM}}=S U(3)_{\mathrm{C}} \times S U(2)_{\mathrm{L}} \times U(1)_{Y}$ is the gauge group of the Standard Model (SM), $B$ and $L$ denote baryon and lepton number respectively. Actually, this is the minimal extension of Minimal SUSY SM (MSSM) which is obtained by promoting to local the already existing $U(1)_{B-L}$ global symmetry of the SM. The spontaneous breaking of $U(1)_{B-L}$ requires the introduction of a conjugate pair of Higgs fields $\Phi$ and $\bar{\Phi}$ which may contain the inflaton. Despite its simplicity, $G_{B-L}$ is strongly motivated by the neutrino physics and leptogenesis. Indeed, within $G_{B-L}$ three right-handed neutrinos, $N_{i}^{c}$, are necessary to cancel the $B-L$ gauge anomaly. Subsequently, the breaking of $U(1)_{B-L}$ via the vacuum expectation values (v.e.vs) of $\Phi$ and $\bar{\Phi}$ naturally provides large Majorana masses to the $N_{i}^{c}$,s, which lead to the tiny neutrino masses via the seesaw mechanism. Furthermore, the out-of-equilibrium decay of $N_{i}^{c}$ gives rise to a robust baryogenesis scenario via non-thermal leptogenesis - see, e.g., Refs. [15-18].

The Higgs mechanism can be implemented by higgsflaton at a scale $M$, well below the the reduced Planck scale $m_{\mathrm{P}}$, adopting the following superpotential [21,22]

$$
W=\lambda S\left(\bar{\Phi} \Phi-M^{2} / 2\right) / 2-\lambda^{\prime} S(\bar{\Phi} \Phi)^{2},
$$

where $\lambda, \lambda^{\prime}$ and $M$ are free parameters. Besides $G_{B-L}, W$ respects a continuous $R$ symmetry [21] under which $S$ and $W$ are equally charged whereas the factor $\bar{\Phi} \Phi$ is uncharged. This type of $W$ is 
well-known from the models of standard [21] (for $\lambda^{\prime}=0$ ) or shifted (for $\lambda^{\prime} \neq 0$ ) F-term hybrid inflation which utilizes $S$ as inflaton with the $\bar{\Phi}-\Phi$ system being stabilized at zero, for $\lambda^{\prime}=0$ [21], or even non-zero, for $\lambda^{\prime} \neq 0$ [22], v.e.v during inflation. In the case of HI, though, the roles of fields are interchanged. The inflaton is included in the $\bar{\Phi}-\Phi$ system whereas $S$ is placed at the origin stabilizing the inflationary potential to be positive and breaking SUSY via its F-term. For these reasons it is called [23] "stabilizer" or Goldstino superfield. Its stability w.r.t the inflationary perturbations can be assured if we include the following term [24]

$$
K_{2}=N_{S} \ln \left(1+|S|^{2} / N_{S}\right)
$$

with $0<N_{S}<6$ in the total Kähler potential. $K_{2}$ parameterizes the compact manifold $\mathcal{M}_{S}=$ $S U(2) / U(1)$. Alternative stabilization methods of $S$ are proposed in Ref. [23, 25] whereas such a necessity is evaded if we assume that $S$ is a nilpotent superfield [26] - for inflation with a single chiral superfield see Ref. [27].

In a series of recent papers [16-20], we demonstrate how we can realize HI within SUGRA taking $\lambda^{\prime}=0$ in Eq. (1.1) and assuming the existence a (positive) non-minimal coupling of the higgsflaton to gravity. E.g., In Ref. [15, 16] we adopt exclusively a strong form of this coupling, in Ref. [19,20] we include also a stronger non-minimal kinetic mixing in the inflationary sector and in Ref. [17] we also apply the hypothesis of induced gravity [28-30]. All the HI models above are fully compatible with the present data [2], most of them do not suffer from any problem with perturbative unitarity [16-18], despite the presence of a strong coupling in the $K$ 's, and some of those [18-20] yield observable gravitational waves. Although quite compelling, though, they are relied to Kähler frames which do not enjoy an exact symmetry and so, their predictability is somehow reduced.

In this paper, we attempt to cure the handicap above, abandoning the hypothesis of the non-minimal gravitational coupling of the higgsflaton. In our present proposal, the inflationary period is predominantly attained due to the existence of a pole of order two in the kinetic term of the inflaton, prior to switching to the canonical variables [31-34]. This pole can be traced out in the hyperbolic geometry [35-38] of the internal space. The importance of this kind of pole in achieving inflationary solutions is stressed in Ref. [39,40] and generalized in Ref. [41-43]. The resulting models can be characterized as $\mathrm{T}$ [31, 35] or $\mathrm{E}$ [44, 45] models depending on the shape of their potential expressed in terms of the canonically normalized inflaton. In particular, the potential of $\mathrm{T}$ models features two symmetric plateaus away from the origin whereas that of E models develops just one shoulder. Independently of the details of the potential, both models share common predictions regarding the scalar spectral index $n_{\mathrm{s}}$ and the tensor-to-scalar ratio $r$ as functions of the number $N_{\star}$ of $e$-foldings elapsed after the pivot scale left the inflationary horizon. Indeed, in the small $\alpha$ limit we obtain [2]

$$
n_{\mathrm{s}}=1-2 / N_{\star} \text { and } r=12 \alpha / N_{\star}^{2} .
$$

Trying to realize, within this regime, HI based on the F-terms - for D-term pole HI see Ref. [46] we select one of the following Kähler potentials for the Higgs superfields

$$
K_{(11)^{2}}=-N \ln \left(1-2|\Phi|^{2}\right)\left(1-2|\bar{\Phi}|^{2}\right) \quad \text { or } \quad K_{21}=-N \ln \left(1-|\Phi|^{2}-|\bar{\Phi}|^{2}\right)^{2},
$$

which respect the symmetries of $W$ and parameterize correspondingly the non-compact hyperbolic spaces - cf. Refs. [33, 34, 45, 47]

$$
\mathcal{M}_{(11)^{2}}=(S U(1,1) / U(1))^{2} \quad \text { or } \quad \mathcal{M}_{21}=S U(2,1) /(S U(2) \times U(1)),
$$

with moduli-space scalar curvatures respectively - see Appendix A

$$
\mathcal{R}_{(11)^{2}}=-4 / N \text { and } \mathcal{R}_{21}=-3 / N
$$


The emergent model can be characterized as an extension of the T model based on the quartic potential with $N=3 \alpha / 2$, symbolized henceforth for short as $\mathrm{TM}_{4}$. Although extensively analyzed, $\mathrm{TM}_{4}$ is mostly realized by a gauge singlet superfield with Kähler potential parameterizing [35-37], in Poincaré disc coordinates, the $S U(1,1) / U(1)$ Kähler manifold and not $\mathcal{M}_{(11)^{2}}$. On the other hand, $\mathcal{M}_{21}$ is widely applied in constructing models of Starobinsky-like inflation [9, 32,-34, 44, 45, 48] and it has not been previously employed in building $\mathrm{TM}_{4}$.

Since the $K$ 's in Eq. (1.4) control, besides the inflaton kinetic term, the SUGRA inflationary potential, $V_{\mathrm{HI}}$, too the pole appearing in the kinetic term is generically expected to appear also in $V_{\mathrm{HI}}$. The successful implementation of HI, however, is facilitated eliminating the pole from $V_{\mathrm{HI}}$. This aim can be accomplished by two alternative strategies - cf. Refs. [35, 37]:

- Constraining $N$ in Eq. (1.4) and tuning the coefficient of non-renormalizable term in Eq. (1.1) such that the denominator including the pole in $V_{\mathrm{HI}}$ is (almost) cancelled out. Indeed, fixing $N=1$ and constraining the ratio $\lambda^{\prime} / \lambda$ or, equivalently, $\delta_{\lambda}$ defined as

$$
\lambda^{\prime} / \lambda \simeq 1+\delta_{\lambda}
$$

we obtain inflationary solutions which cover the observationally favored $n_{\mathrm{s}}$ region maintaining $r$ at a low level. Variation of $M$ as a function of $\delta_{\lambda}$ does not disrupt the realization of HI.

- Complicating the Kähler potentials so that they influence just the kinetic part of the inflationary sector and not at all $V_{\mathrm{HI}}$. In particular, we may adopt the following $K$ 's

$$
\widetilde{K}_{(11)^{2}}=-N \ln \frac{\left(1-2|\Phi|^{2}\right)\left(1-2|\bar{\Phi}|^{2}\right)}{(1-2 \bar{\Phi} \Phi)\left(1-2 \bar{\Phi}^{*} \Phi^{*}\right)} \text { or } \quad \widetilde{K}_{21}=-N \ln \frac{\left(1-|\Phi|^{2}-|\bar{\Phi}|^{2}\right)^{2}}{(1-2 \bar{\Phi} \Phi)\left(1-2 \bar{\Phi}^{*} \Phi^{*}\right)}
$$

which yield the same lagrangian kinetic terms and share the same curvatures with $K_{(11)^{2}}$ and $K_{21}$ respectively. It is obvious, though, that along the inflationary path defined by the condition $|\Phi|=|\bar{\Phi}|$, the $\widetilde{K}$ 's above, considered as exponents of the exponential factor entering the SUGRA scalar potential - see Sec. 2 below -, reduce to identity and so, the expression of $V_{\mathrm{HI}}$ coincides with its SUSY version. In these cases, the nonrenormalizable part of $W$ in Eq. (1.1) is totally irrelevant whereas $N$ may be handled as a free parameter yielding thereby, in its large regime, $r$ close to its upper bound [2]. Note, in passing, that $K$ similar to $\widetilde{K}_{(11)^{2}}$ is already employed in Ref. [49] within a scenario where $U(1)_{B-L}$ is replaced by a global $U(1)$ Peccei-Quinn symmetry.

Summarizing, in this paper we analyze two novel models of HI, MI and MII, employing $W$ in Eq. (1.1) in conjunction with $K_{2}$ in Eq. (1.2) and the "untilded" or the "tilded" $K$ 's in Eqs. (1.4) and (1.8) respectively. More specifically, MI and MII are defined as follows

MI: $K=K_{2(11)^{2}}=K_{2}+K_{(11)^{2}}$ or $K=K_{221}=K_{2}+K_{21}$ with $N=1$ and $W$ with $\lambda^{\prime} \neq 0$; MII: $K=\widetilde{K}_{2(11)^{2}}=K_{2}+\widetilde{K}_{(11)^{2}}$ or $K=\widetilde{K}_{221}=K_{2}+\widetilde{K}_{21}$ with free $N$ and $W$ with $\lambda^{\prime}=0$.

In both models $M$ in Eq. (1.1) remains a free parameter which may be constrained by the gauge coupling unification within MSSM.

The rest of the paper is organized as follows: In Sec. 2 we shortly review the derivation of the potential employed for $B-L$ HI within SUGRA. In Sec. 3 our inflationary models are confronted with observations. Our conclusions are summarized in Sec. 4. Some mathematical notions related to the geometric structure of the Kähler manifolds encountered in our set-up are exhibited in Appendix A. Throughout, the complex scalar components of the various superfields are denoted by the same superfield symbol, charge conjugation is denoted by a star $\left(^{*}\right)$ - e.g., $|Z|^{2}=Z Z^{*}$ - the symbol, $Z$ as subscript denotes derivation with respect to (w.r.t) $Z$, and we use units where the reduced Planck scale $m_{\mathrm{P}}=2.43 \cdot 10^{18} \mathrm{GeV}$ is equal to unity. 


\section{Supergravity FrameWORK}

In Sec. 2.1 we describe the embedding of $B-L$ HI within SUGRA, and then, in Sec. 2.2, we determine the canonically normalized fields which are involved in our scenario. In Sec. 2.3, we derive the inflationary potential of our models in the tree level and check its stability in Sec. 2.4. For presentation purposes, the formulae of this section is derived for an unspecified $N$ value, even for MI where we set $N=1$ at last.

\subsection{General Framework}

The part of the Einstein-frame action within SUGRA related to the complex scalars $Z^{I}=S, \bar{\Phi}, \Phi$ - which are involved in the $W$ and $K$ 's of our models as defined in Eq. (1.9) - has the form [52]

$$
\mathrm{S}=\int d^{4} x \sqrt{-\mathfrak{g}}\left(-\frac{1}{2} \mathcal{R}+G_{I \bar{J}} g^{\mu \nu} D_{\mu} Z^{I} D_{\nu} Z^{* \bar{J}}-V\right)
$$

where $\mathcal{R}$ is the space-time Ricci scalar curvature, $\mathfrak{g}$ is the determinant of the background FriedmannRobertson-Walker metric, $g^{\mu \nu}$ with signature $(+,-,-,-)$ and $G$ with

$$
G\left(Z^{I}, Z^{* \bar{J}}\right)=K\left(Z^{I}, Z^{* \bar{J}}\right)+\ln \left|W\left(Z^{I}\right)\right|^{2}
$$

is the Kähler -invariant function, subject to the notation

$$
G_{I \bar{J}}=G_{, Z^{I} Z^{* \bar{J}}}=K_{I \bar{J}} \text { and } G^{I \bar{J}} G_{L \bar{J}}=\delta_{L}^{I} .
$$

Also, $D_{\mu}$ is the gauge covariant derivative which operates on the fields $Z^{I}$ as follows

$$
D_{\mu} S=\partial_{\mu} S, \quad D_{\mu} \Phi=\partial_{\mu} \Phi+i g A_{B L \mu} \Phi \text { and } D_{\mu} \bar{\Phi}=\partial_{\mu} \bar{\Phi}-i g A_{B L \mu} \bar{\Phi},
$$

where $A_{B L \mu}$ is the $U(1)_{B-L}$ gauge field with an associated gauge coupling constant $g$. Here we take into account that $S$ is a $U(1)_{B-L}$ singlet whereas the $\Phi$ and $\bar{\Phi}$ are the $U(1)_{B-L}$ Higgs fields with $(B-L)$ charges 1 and -1 respectively.

Finally, $V$ in Eq. (2.1) is the SUGRA potential which includes the contributions $V_{\mathrm{F}}$ and $V_{\mathrm{D}}$ from $\mathrm{F}$ and $\mathrm{D}$ terms respectively. It can be calculated via the formula

$$
V=V_{\mathrm{F}}+V_{\mathrm{D}} \text { with } V_{\mathrm{F}}=e^{K}\left(K^{I \bar{J}} D_{I} W D_{\bar{J}}^{*} W^{*}-3|W|^{2}\right) \text { and } V_{\mathrm{D}}=\frac{g^{2}}{2} \mathrm{D}_{B L}^{2},
$$

where $D_{I}$ is the Kähler-covariant derivative and $\mathrm{D}_{B L}$ is the $\mathrm{D}$ term corresponding to $U(1)_{B-L}$. Also, we use the shorthand notation

$$
K^{I \bar{J}} K_{I \bar{L}}=\delta_{\bar{L}}^{\bar{J}}, D_{I} W=W_{, Z^{I}}+K_{, Z^{I}} W \text { and } \mathrm{D}_{B L}=Z_{I}(B-L) K^{I} \text { with } K^{I}=K_{, Z^{I}} .
$$

In $V_{\mathrm{D}}$ we neglect any contribution from Fayet-Iliopoulos terms and we adopt a trivial gauge kinetic function. For the $K$ 's in Eqs. (1.4) and (1.8) $\mathrm{D}_{B L}$ takes the form

$$
\mathrm{D}_{B L}=2 N\left(|\Phi|^{2}-|\bar{\Phi}|^{2}\right) \cdot \begin{cases}\left(1-2|\bar{\Phi}|^{2}\right)^{-1}\left(1-2|\bar{\Phi}|^{2}\right)^{-1} & \text { for } K=K_{(11)}, \widetilde{K}_{(11)^{2}}, \\ \left(1-|\Phi|^{2}-|\bar{\Phi}|^{2}\right)^{-1} & \text { for } K=K_{21}, \widetilde{K}_{21} .\end{cases}
$$

From this result we deduce that $V_{\mathrm{D}}$ can be eliminated during HI if $|\Phi|=|\bar{\Phi}|$, i.e., if we identify inflaton with the radial parts of $\Phi$ and $\bar{\Phi}$. More explicitly, we parameterize the fields of our models as follows [15-20]

$$
\Phi=\phi e^{i \theta} \cos \theta_{\Phi}, \quad \bar{\Phi}=\phi e^{i \bar{\theta}} \sin \theta_{\Phi} \quad \text { with } 0 \leq \theta_{\Phi} \leq \pi / 2 \text { and } S=(s+i \bar{s}) / \sqrt{2}
$$

and investigate the implementation of HI driven by the real field $\phi$ along the field configuration

$$
\bar{s}=s=\bar{\theta}=\theta=0 \text { and } \theta_{\Phi}=\pi / 4 .
$$

This selection ensures the $\mathrm{D}$ flatness, since $\mathrm{D}_{B L}=0-\mathrm{cf}$. Ref. [46] -, and the avoidance of a possible runaway problem, since the term $-3|W|^{2}$ in Eq. (2.4) vanishes thanks to the constraint $S=0$ [23]. 


\subsection{Canonically Normalized Fileds}

To specify the canonically normalized fields, we note that, for all choices of $K$ in Eqs. (1.4) and (1.8), $K_{I \bar{J}}$ along the configuration in Eq. (2.7) takes the form

$$
\left(K_{I \bar{J}}\right)=\operatorname{diag}\left(M_{\bar{\Phi} \Phi}, K_{S S^{*}}\right)
$$

where $K_{S S^{*}}=K_{2 S S^{*}}=1$ and $M_{\bar{\Phi} \Phi}=\left(K_{I \bar{J}}\right)$ is the matrix containing the elements of $K_{I \bar{J}}$ with $I=\bar{\Phi}, \Phi$. It is found to be

$$
M_{\bar{\Phi} \Phi}=\left\{\begin{array} { l l } 
{ \kappa \operatorname { d i a g } ( 1 , 1 ) } & { \text { for } K = K _ { ( 1 1 ) ^ { 2 } } , \widetilde { K } _ { ( 1 1 ) ^ { 2 } } , } \\
{ ( \kappa \phi ^ { 2 } / 2 ) } & { \text { for } K = K _ { 2 1 } , \widetilde { K } _ { 2 1 } , } \\
{ 1 } & { 2 / \phi ^ { 2 } - 1 }
\end{array} \quad \text { with } \left\{\begin{array}{l}
\kappa=2 N / f_{K}^{2} \\
\text { and } \\
f_{K}=1-\phi^{2}
\end{array}\right.\right.
$$

We observe that $M_{\bar{\Phi} \Phi}$ is diagonal for $K=K_{(11)^{2}}$ and $\widetilde{K}_{(11)^{2}}$ whereas it requires diagonalization for $K=K_{21}$ and $\widetilde{K}_{21}$. In the latter case its eigenvalues are

$$
\kappa_{+}=\kappa \text { and } \kappa_{-}=\kappa f_{K}
$$

The equality of the kinetic terms for "tilded" and "untilded" $K$ 's with the same arithmetic indices can be understood by the observation that the structure of the "tilded" $K$ 's is

$$
\widetilde{K}_{(i j)}\left(\bar{\Phi}, \bar{\Phi}^{*}, \Phi, \Phi^{*}\right)=K_{(i j)}\left(\bar{\Phi}, \bar{\Phi}^{*}, \Phi, \Phi^{*}\right)+K_{\mathrm{H}}(\bar{\Phi}, \Phi)+K_{\mathrm{A}}\left(\bar{\Phi}^{*}, \Phi^{*}\right)
$$

where $(i j)=(11)^{2}$ or $(i j)=21$ and the subscripts "H" and "A" stand for "holomorphic" and "antiholomorphic" respectively. More explicitly, comparing Eq. (2.11) with Eqs. (1.4) and (1.8) we infer

$$
K_{\mathrm{H}}=N \ln (1-2 \bar{\Phi} \Phi) \text { and } K_{\mathrm{A}}=N \ln \left(1-2 \bar{\Phi}^{*} \Phi^{*}\right) \text {. }
$$

Therefore, the mixed derivatives of $\widetilde{K}_{(i j)}$ coincide with the corresponding ones of $K_{(i j)}$ since $\partial_{\bar{I}} K_{\mathrm{H}}=$ $\partial_{I} K_{\mathrm{A}}=0$ for $I=\bar{\Phi}, \Phi$. As a further consequence, the Kähler metrics $K_{I \bar{J}}$ and $\widetilde{K}_{I \bar{J}}$ for $K=K_{(i j)}$ and $\widetilde{K}=\widetilde{K}_{(i j)}$ with fixed $(i j)$ turn out to be equal.

Expanding the second term of the right-hand side (r.h.s) of Eq. (2.1) along the path in Eq. (2.7) for $I=\Phi, \bar{\Phi}$ and substituting there Eq. (2.9), we obtain

$$
K_{I \bar{J}} \dot{Z}^{I} \dot{Z}^{* \bar{J}}= \begin{cases}\kappa \dot{\phi}^{2}+\kappa \phi^{2}\left(\dot{\theta}_{+}^{2}+\dot{\theta}_{-}^{2}+2 \dot{\theta}_{\Phi}^{2}\right) / 2 & \text { for } K=K_{(11)^{2}}, \widetilde{K}_{(11)^{2}}, \\ \kappa_{+}\left(\dot{\phi}^{2}+\phi^{2} \dot{\theta}_{+}^{2} / 2\right)+\kappa_{-} \phi^{2}\left(\dot{\theta}_{-}^{2} / 2+\dot{\theta}_{\Phi}^{2}\right) & \text { for } K=K_{21}, \widetilde{K}_{21},\end{cases}
$$

where $\theta_{ \pm}=(\bar{\theta} \pm \theta) / \sqrt{2}$ and the dot denotes derivation w.r.t the cosmic time, $t$. Comparing the expressions above with the following one

$$
K_{I \bar{J}} \dot{Z}^{I} \dot{Z}^{* \bar{J}} \simeq \frac{1}{2}\left(\dot{\hat{\phi}}^{2}+\dot{\hat{\theta}}_{+}^{2}+\dot{\hat{\theta}}_{-}^{2}+\dot{\hat{\theta}}_{\Phi}^{2}\right)
$$

we can specify the canonically normalized fields, denoted by hat, in terms of the initial (unhatted) ones - recall that $S$ is already cationically normalized. Thanks to Eqs. 2.9) and (2.10) we can establish for all $K$ 's a unique expression regarding the normalized inflaton, i.e.,

$$
d \widehat{\phi} / d \phi=J=2 \sqrt{N} / f_{K},
$$


from which we infer that the kinetic term of $\phi$ exhibits a pole at $\phi=1$ of order two - see $f_{K}$ in Eq. (2.9). For the remaining fields of the $\bar{\Phi}-\Phi$ system we find

$$
\begin{array}{ll}
\widehat{\theta}_{ \pm}=\sqrt{\kappa} \phi \theta_{ \pm}, \widehat{\theta}_{\Phi}=\sqrt{2 \kappa} \phi\left(\theta_{\Phi}-\pi / 4\right) & \text { for } K=K_{(11)^{2}}, \widetilde{K}_{(11) 2} \\
\widehat{\theta}_{+}=\sqrt{\kappa_{+}} \phi \theta_{+}, \widehat{\theta}_{-}=\sqrt{\kappa_{-}} \phi \theta_{-}, \widehat{\theta}_{\Phi}=\sqrt{2 \kappa_{-}} \phi\left(\theta_{\Phi}-\pi / 4\right) & \text { for } K=K_{21}, \widetilde{K}_{21} .
\end{array}
$$

Integrating Eq. (2.13c) we can identify $\widehat{\phi}$ in terms of $\phi$, as follows

$$
\widehat{\phi}=\sqrt{N} \ln \frac{1+\phi}{1-\phi} \Rightarrow \phi=\tanh \frac{\widehat{\phi}}{2 \sqrt{N}},
$$

where we set the relevant constant of integration equal to zero. We can easily conclude that for $0 \leq$ $\phi \leq 0.999, \widehat{\phi}$ gets increased from 0 to $7.6 \sqrt{N}$, i.e., $\widehat{\phi}$ can be much larger than $\phi$ facilitating thereby the attainment of HI (which is actually of chaotic type and necessitates $\widehat{\phi} \gg 1$ ) with subplanckian $\phi$ 's. The last fact is imperative for a meaningful approach to SUGRA. Indeed, terms of the form $(\Phi \bar{\Phi})^{p}$ with $p>2$ in $W$ are not disallowed by the symmetries and so stabilization of our scenario against corrections from those $W$ terms dictates subplanckian values for $\bar{\Phi}$ and $\Phi$ or, via Eq. (2.6), $\phi$.

\subsection{Inflationary Potential}

Along the trough in Eq. (2.7) the only surviving term in Eq. (2.4) is

$$
V_{\mathrm{HI}}=e^{K} K^{S S^{*}}\left|W_{, S}\right|^{2} \text {. }
$$

Taking into account Eqs. (1.2) and (1.9), we obtain $K^{S S^{*}}=1 / K_{2 S S^{*}}=\left.\left(1+|S|^{2} / N_{S}\right)^{2}\right|_{S=0}=1$. Also, Eqs. (1.4) and (1.8) yield

$$
e^{K}=\left\{\begin{array}{lll}
f_{K}^{-2 N} & \text { for } K=K_{(11)^{2}} \text { and } & K_{21} \\
1 & \text { for } K=\widetilde{K}_{(11)^{2}} \text { and } \widetilde{K}_{21}
\end{array}\right.
$$

i.e., the pole in $f_{K}$ is presumably present in $V_{\mathrm{HI}}$ of $\mathrm{MI}$, but it disappears in $V_{\mathrm{HI}}$ of $\mathrm{MII}$, as anticipated below Eq. (1.8). Substituting the results above and Eq. (1.1) into Eq. (2.15a), this takes its master form

$$
V_{\mathrm{HI}}=\frac{\lambda^{2}}{16} \cdot \begin{cases}\left(\phi^{2}-\left(1+\delta_{\lambda}\right) \phi^{4}-M^{2}\right)^{2} / f_{K}^{2 N} & \text { for MI, } \\ \left(\phi^{2}-M^{2}\right)^{2} & \text { for MII . }\end{cases}
$$

From the first equation above we easily infer that the elimination of the pole from the denominator of $V_{\mathrm{HI}}$ can be implemented as $M$ and $\delta_{\lambda}$ tend to zero only for $N=1$. No $N$ dependence arises for MII.

To directly compare our models with the $\mathrm{TM}_{4}[2,35]$, we express $V_{\mathrm{HI}}$ in Eq. (2.16) as a function of $\widehat{\phi}$, taking advantage of Eq. (2.14). We find

$$
V_{\mathrm{HI}}=\frac{\lambda^{2}}{16} \cdot \begin{cases}\cosh ^{4 N}(\widehat{\phi} / 2 \sqrt{N})\left(\tanh ^{2}(\widehat{\phi} / 2 \sqrt{N})-\left(1+\delta_{\lambda}\right) \tanh ^{4}(\widehat{\phi} / 2 \sqrt{N})-M^{2}\right)^{2} & \text { for MI } \\ \left(\tanh ^{2}(\widehat{\phi} / 2 \sqrt{N})-M^{2}\right)^{2} & \text { for MII } .\end{cases}
$$

From the expressions above we conclude that MI for $N=1$ and $M=\delta_{\lambda}=0$ and MII for $M=0$ share the same expression for $V_{\mathrm{HI}}$, which is actually the potential adopted for $\mathrm{TM}_{4}$ [31, 35], i.e.

$$
V_{\mathrm{HIO}}=\lambda^{2} \phi^{4} / 16=\lambda^{2} \tanh ^{4}(\widehat{\phi} / 2) / 16 .
$$

The naive expectation that this model is observationally ruled out by now [2] since $V_{\mathrm{HI}}=V_{\mathrm{HI} 0}(\phi)$ coincides with the one of the quartic power-low model is not correct, since $\phi$ is not canonically normalized. This is related to $\widehat{\phi}$ via Eq. (2.14) and so, the emerging picture is radically different. Indeed, 


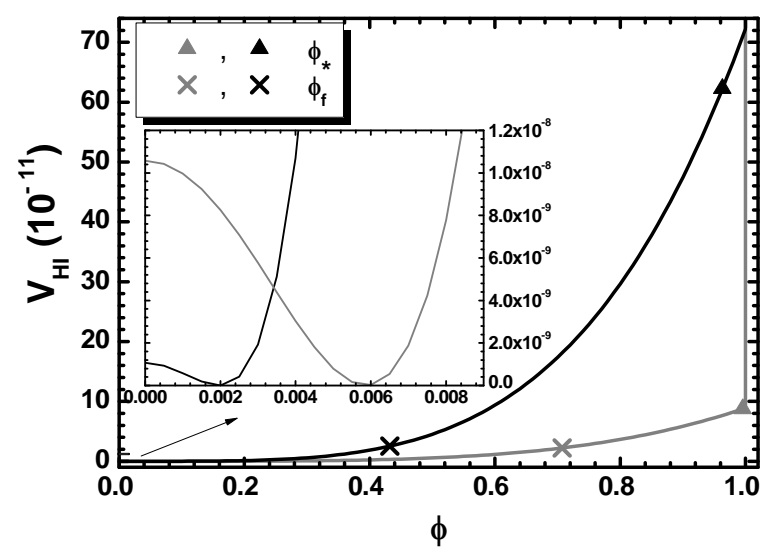

(a)

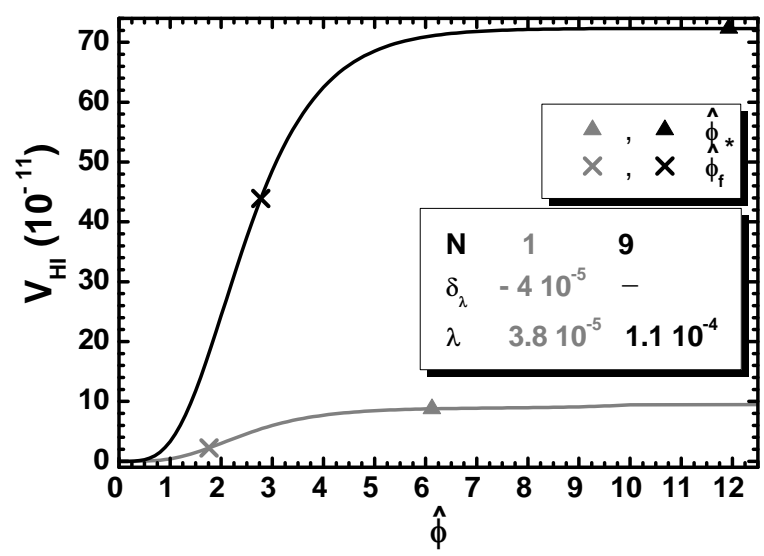

(b)

Figure 1: Inflationary potential $V_{\mathrm{HI}}$ as a function of (a) $\phi$ for $\phi>0$ and (b) $\widehat{\phi}$ for $\widehat{\phi}>0$ fixing $M_{B L}=M_{\mathrm{GUT}}$. We consider MI with $\delta_{\lambda}=-4 \cdot 10^{-5}$ and $\lambda=3.8 \cdot 10^{-5}$ (gray lines) or MII with $N=9$ and $\lambda=1.1 \cdot 10^{-4}$ (black lines). Values corresponding to $\phi_{\star}$ and $\phi_{\mathrm{f}}$ (a) or $\widehat{\phi}$ and $\widehat{\phi}_{\mathrm{f}}$ (b) are depicted. Shown is also the low- $\phi$ behavior of $V_{\mathrm{HI}}$ in the inset (a).

$V_{\mathrm{HIO}}=V_{\mathrm{HI}}(\widehat{\phi})$ in Eq. (2.18) is encountered in $\mathrm{TM}_{4}$ which, although constrained, is still observationally [2] alive.

To clarify further this key feature of our models, we comparatively plot $V_{\mathrm{HI}}$ as a function of $\phi$ in Fig. 11-(a) and $\widehat{\phi}$ in Fig. 1 - b) for MI (gray lines) and MII (black lines) - cf. Ref. [31]. Here we impose the constraint of MSSM gauge coupling unification which entails $M=5.87 \cdot 10^{-3}$ for MI and $M=1.96 \cdot 10^{-3}$ for MII - see Sec.3.1.2 and 3.3. Observational constraints determine $\lambda$ and $\delta_{\lambda}$ for MI or $\lambda$ and $N$ for MII. Their values are shown in the legend of Fig. 11-(b). We notice in Fig. 11.(a) that $V_{\mathrm{HI}}$ for both models have a parabolic-like slope for $\phi<1$ whereas MI exhibits a sharp increase as $\phi$ tends to 1 due to the pole - see Eq. (2.16). On the contrary, in Fig. 1-(b) $V_{\mathrm{HI}}$ experiences a stretching for $\widehat{\phi}>1$ which results to a plateau facilitating, thereby, the establishment of $\mathrm{HI}$ for $\widehat{\phi} \gg 1$. We remark that a gap of about one order of magnitude emerges between $V_{\mathrm{HI}}=V_{\mathrm{HI}}(\widehat{\phi})$ for MI and MII which has as a consequence the elevation - see Sec. 3.3- of $r$ as explained in Ref. [50]. The two crucial values of $\phi$ depicted in Fig. 1. (a), $\phi_{\mathrm{f}}$ and $\phi_{\star}$, which limit the observationally relevant inflationary period see Sec. 3.1.1 - are 0.71 and 0.99564 for MI or 0.43 and 0.96322 for MII. These values are enhanced according to Eq. (2.14), i.e., $\widehat{\phi}_{\mathrm{f}}=1.763$ and $\widehat{\phi}_{\star}=6.12$ for MI $\widehat{\phi}_{\mathrm{f}}=2.77$ and $\widehat{\phi}_{\star}=11.93$ for MII see Fig. 1-(b). The structure of $V_{\mathrm{HI}}$ for low $\phi$ values, responsible for the implementation of the Higgs mechanism, is also illustrated in the inset of Fig.1-(a). Similar behavior is expected for $V_{\mathrm{HI}}=V_{\mathrm{HI}}(\widehat{\phi})$.

\subsection{STABILITY AND LOOP-CORRECTIONS}

To consolidate our inflationary models, we have to verify that the inflationary direction in Eq. 2.7) is stable w.r.t the fluctuations of the non-inflaton fields. To this end, we construct the mass-squared spectrum of the various scalars defined in Eq. (2.13d). Taking $\delta_{\lambda} \simeq 0$ and $M \ll \phi$ we find the expressions of the masses squared $\widehat{m}_{\chi^{\alpha}}^{2}$ (with $\chi^{\alpha}=\theta_{+}, \theta_{\Phi}$ and $S$ ) and arrange them in Table 1 . These masses are compared with the Hubble parameter during HI, $H_{\mathrm{HI}}^{2}=V_{\mathrm{HI}} / 3$. For $\phi \simeq \phi_{\star} \simeq 1$ the exposed formulas fairly approach the quite lengthy, exact expressions taken into account in our numerical computation. From our findings in Table 1 we easily confirm that $\widehat{m}_{\chi^{\alpha}}^{2} \gg H_{\mathrm{HI}}^{2}$ for $\chi^{\alpha}=\theta_{+}$ and $\theta_{\Phi}-$ note that $g \gg \lambda$. As regards the crucial stabilization of $S$, the negative contributions of the $N$-dependent terms are maximized for $N=1$ and both MI and MII, and so we deduce that the 


\begin{tabular}{|c|c|c|c|c|c|c|}
\hline \multirow[t]{2}{*}{ FIELDS } & \multirow{2}{*}{$\begin{array}{l}\text { EIGEN- } \\
\text { STATES } \\
\end{array}$} & \multicolumn{5}{|c|}{ MASSES SQUARED } \\
\hline & & & $K=K_{2(11)^{2}}$ & $K=K_{221}$ & $K=\widetilde{K}_{2(11)^{2}}$ & $K=\widetilde{K}_{221}$ \\
\hline \multirow{3}{*}{$\begin{array}{l}2 \text { real } \\
\text { scalars }\end{array}$} & \multirow{3}{*}{$\begin{array}{l}\widehat{\theta}_{+} \\
\widehat{\theta}_{\Phi}\end{array}$} & \multirow{3}{*}{$\begin{array}{l}m_{\widehat{\theta}+}^{2} \\
\widehat{m}_{\theta_{\Phi}}^{2}\end{array}$} & \multicolumn{4}{|c|}{$3 H_{\mathrm{HI}}^{2}$} \\
\hline & & & \multirow{2}{*}{$\begin{array}{l}M_{B L}^{2}+6 H_{\mathrm{HI}}^{2}(1+2 / N \\
\left.-1 / N \phi^{2}-\phi^{2} / N\right)\end{array}$} & \multirow{2}{*}{$\begin{array}{l}M_{B L}^{2}+6 H_{\mathrm{HI}}^{2}(1 \\
\left.+1 / N-1 / N \phi^{2}\right) \\
\end{array}$} & \multicolumn{2}{|c|}{ As for } \\
\hline & & & & & $K=K_{2(11)^{2}}$ & $K=K_{221}$ \\
\hline $\begin{array}{l}1 \text { complex } \\
\text { scalar }\end{array}$ & $s, \bar{s}$ & $\widehat{m}_{s}^{2}$ & \multicolumn{2}{|c|}{$\begin{array}{c}6 H_{\mathrm{HI}}^{2}\left(1 / N_{S}-4\left(1-\phi^{2}\right) / N+N \phi^{2}\right. \\
\left.+2\left(1-2 \phi^{2}\right)+4 \phi^{2} / N\right) \\
\end{array}$} & \multicolumn{2}{|c|}{$\begin{array}{r}6 H_{\mathrm{HI}}^{2}\left(1 / N_{S}-2 / N\right. \\
\left.+1 / N \phi^{2}+\phi^{2} / N\right) \\
\end{array}$} \\
\hline $\begin{array}{l}1 \text { gauge } \\
\text { boson }\end{array}$ & $A_{B L}$ & $M_{B L}^{2}$ & \multicolumn{4}{|c|}{$4 N g^{2} \phi^{2} / f_{K}^{2}$} \\
\hline \multirow{2}{*}{$\begin{array}{l}4 \text { Weyl } \\
\text { spinors }\end{array}$} & \multirow{2}{*}{$\begin{array}{c}\widehat{\psi}_{ \pm} \\
\lambda_{B L}, \widehat{\psi}_{\Phi-}\end{array}$} & $\widehat{m}_{\psi \pm}^{2}$ & \multicolumn{4}{|c|}{$3 f_{K}^{2} H_{\mathrm{HI}}^{2} / N^{2} \phi^{2}$} \\
\hline & & $M_{B L}^{2}$ & \multicolumn{4}{|c|}{$4 N g^{2} \phi^{2} / f_{K}^{2}$} \\
\hline
\end{tabular}

Table 1: The mass-squared spectra along the inflationary trajectory of Eq. (2.7) for various $K$ 's and $\phi \lesssim 1$. To avoid very lengthy formulas we set $\delta_{\lambda} \simeq 0$ and neglect terms proportional to $M \ll \phi$.

minimal value of $\widehat{m}_{s}^{2}\left(\phi_{\star}\right)$ is $\left.\widehat{m}_{s}^{2}\left(\phi_{\star}\right)\right|_{\min } \simeq 6 H_{\mathrm{HI}}^{2} / N_{S}$ which stays positive and heavy enough for $0<N_{S}<6$. As $\phi$ decreases towards the SUSY vacuum, $\widehat{m}_{s}^{2}$ increases, thanks to the term with $\phi$ in the denominators. For MII, as $N$ increases $m_{s}^{2}$ increases too since the negative contributions with $N$ in the denominators decrease. In Table 1 we also display the masses, $M_{B L}$, of the gauge boson $A_{B L}$ - which signals the fact that $U(1)_{B-L}$ is broken (already) during $\mathrm{HI}$ - and the masses of the corresponding fermions. Note that the unspecified eigestate $\widehat{\psi}_{ \pm}$is defined as

$$
\widehat{\psi}_{ \pm}=\left(\widehat{\psi}_{\Phi+} \pm \widehat{\psi}_{S}\right) / \sqrt{2} \text { with } \psi_{\Phi \pm}=\left(\psi_{\Phi} \pm \psi_{\bar{\Phi}}\right) / \sqrt{2}
$$

with the spinors $\psi_{S}$ and $\psi_{\Phi \pm}$ being associated with the superfields $S$ and $\bar{\Phi}-\Phi$. The broken generator of $B-L$ leads to one would-be-Goldstone boson $\theta_{-}$, "eaten" by the gauge boson $A_{B L}$ which becomes massive. If we take into account $\phi$, not included in Table 1, we can easily verify that the degrees of freedom of the particle spectrum before and after the spontaneous breaking of $B-L$ are equal. Similarly, the fermionic and bosonic degrees of freedom are also equal - cf. Ref. [19]. As a byproduct of the fact that $U(1)_{B-L}$ is already broken during $\mathrm{HI}$, no cosmic string are produced at the end of $\mathrm{HI}$ - contrary to what happens within standard F-term hybrid inflation [21].

The derived mass spectrum can be employed in order to find the one-loop radiative corrections, $\Delta V_{\mathrm{HI}}$, to $V_{\mathrm{HI}}$. Considering SUGRA as an effective theory with cutoff scale equal to $m_{\mathrm{P}}$, the wellknown Coleman-Weinberg formula - cf. Ref. [15, 16, 18, 19,28] - can be employed taking into account only the masses which lie well below $m_{\mathrm{P}}$, i.e., all the masses arranged in Table 1 besides $M_{B L}$ and $\widehat{m}_{\theta_{\Phi}}$. The resulting $\Delta V_{\mathrm{HI}}$ leaves intact our inflationary outputs, provided that the renormalizationgroup mass scale $Q$, is determined by requiring $\Delta V_{\mathrm{HI}}\left(\phi_{\star}\right)=0$ or $\Delta V_{\mathrm{HI}}\left(\phi_{\mathrm{f}}\right)=0$, where $\phi_{\star}$ and $\phi_{\mathrm{f}}$ are the observationally relevant values of $\phi-$ see Sec. 3.1.1. The first of the conditions above - the latter leads to similar $Q$ values [19] - yields $Q \simeq(1.1-1.4) \cdot 10^{-5}$ for MI or $Q \simeq(1.2-6.3) \cdot 10^{-5}$ for MII and the values of parameters given in Eqs. (3.24) and (3.25) below. This determination of $Q$ renders our results practically independent of $Q$ since these can be derived exclusively by using $V_{\mathrm{HI}}$ in Eq. (2.16) with the various quantities evaluated at $Q$. Note that their renormalization-group running is expected to be negligible because $Q$ is close to the inflationary scale $V_{\mathrm{HI} \star}^{1 / 4} \simeq(2.8-3.2) \cdot 10^{-3}$ for MI or $V_{\mathrm{HI} \star}^{1 / 4} \simeq(2.98-6.8) \cdot 10^{-3}$ for MII corresponding to Hubble parameters $H_{\mathrm{HI} \star} \simeq(4.5-5.9) \cdot 10^{-6}$ for MI or $H_{\mathrm{HI} \star} \simeq(0.5-2.5) \cdot 10^{-5}$ for MII - all the quantities are computed for $\phi=\phi_{\star}$. 


\section{INFLATION ANALYSIS}

In Secs. 3.2 and 3.3 below we ascertain analytically and numerically respectively, if the $V_{\mathrm{HI}}$ in Eq. (2.16) endowed with the $\phi$ normalization in Eq. (2.13c) may be consistent with a number of constraints introduced in Sec. 3.1 .

\subsection{General FrameWORK}

The constraints imposed on our inflationary setting may be grouped in two categories (observational and theoretical) which are described respectively in Secs. 3.1.1 and 3.1.2 below.

\subsubsection{Observational Constraints}

(a) Number of e-foldings and Normalization of the Power Spectrum. The number of e-foldings $N_{\star}$ that the scale $k_{\star}=0.05 / \mathrm{Mpc}$ experiences during $\mathrm{HI}$ and the amplitude $A_{\mathrm{S}}$ of the power spectrum of the curvature perturbations generated by $\phi$ can be computed using the standard formulae [8]

$$
N_{\star}=\int_{\widehat{\phi}_{\mathrm{f}}}^{\widehat{\phi}_{\star}} d \widehat{\phi} \frac{V_{\mathrm{HI}}}{V_{\mathrm{HI}, \widehat{\phi}}} \text { and } A_{\mathrm{s}}^{1 / 2}=\frac{1}{2 \sqrt{3} \pi} \frac{V_{\mathrm{HI}}^{3 / 2}\left(\widehat{\phi}_{\star}\right)}{\left|V_{\mathrm{HI}, \widehat{\phi}}\left(\widehat{\phi}_{\star}\right)\right|},
$$

where $\widehat{\phi}_{\star}$ is the value of $\widehat{\phi}$ when $k_{\star}$ crosses outside the inflationary horizon, and $\widehat{\phi}_{\mathrm{f}}$ is the value of $\widehat{\phi}$ at the end of HI, which can be found, in the slow-roll approximation, from the condition

$$
\max \{\epsilon(\widehat{\phi}),|\eta(\widehat{\phi})|\} \simeq 1 \text {, where } \epsilon=\frac{1}{2}\left(\frac{V_{\mathrm{HI}, \widehat{\phi}}}{V_{\mathrm{HI}}}\right)^{2} \text { and } \eta=\frac{V_{\mathrm{HI}, \widehat{\phi} \widehat{\phi}}}{V_{\mathrm{HI}}}
$$

The observables above are to be confronted with the [1]

$$
N_{\star} \simeq 61.5+\frac{1}{4} \ln \frac{V_{\mathrm{HI}}\left(\widehat{\phi}_{\star}\right)^{2}}{g_{\mathrm{rh} *}^{1 / 3} V_{\mathrm{HI}}\left(\widehat{\phi}_{\mathrm{f}}\right)} \text { and } \sqrt{A_{\mathrm{s}}} \simeq 4.588 \cdot 10^{-5},
$$

where for the latter restriction we take into account the final full-mission Planck measurements (TT, TE, EE+lowE+lensing) and Baryon Acoustic Oscillations (BAO) dataset. Also, we assumed that HI is followed in turn by a oscillatory phase with mean equation-of-state parameter $w_{\mathrm{rh}} \simeq 1 / 3$ - which corresponds to a quatric potential [2] -, radiation and matter domination, $T_{\mathrm{rh}}$ is temperature after HI, $g_{\mathrm{rh} *}$ is the energy-density effective number of degrees of freedom at the reheat temperature $T_{\mathrm{rh}}-$ for the MSSM spectrum we take $g_{\mathrm{rh} *}=228.75$. We observe that $N_{\star}$ turns out to be independent of the explicit value of $T_{\mathrm{rh}}$.

(b) Remaining Observables. The remaining inflationary observables (the spectral index $n_{\mathrm{s}}$, its running $a_{\mathrm{S}}$, and the tensor-to-scalar ratio $r$ ) are estimated through the relations:

$$
n_{\mathrm{s}}=1-6 \epsilon_{\star}+2 \eta_{\star}, \quad a_{\mathrm{s}}=\frac{2}{3}\left(4 \eta_{\star}^{2}-\left(n_{\mathrm{s}}-1\right)^{2}\right)-2 \xi_{\star} \text { and } r=16 \epsilon_{\star},
$$

where $\xi=V_{\mathrm{HI}, \widehat{\phi}} V_{\mathrm{HI}, \widehat{\phi} \widehat{\phi} \hat{\phi}} / V_{\mathrm{HI}}^{2}$ and the variables with subscript $\star$ are evaluated at $\phi=\phi_{\star}$. These observables must be in agreement with the fitting of the Planck TT, TE, EE+lowE+lensing, BK14 and BAO data [2,3] with $\Lambda \mathrm{CDM}+r$ model which approximately requires at $95 \%$ confidence level (c.I.)

$$
n_{\mathrm{S}}=0.967 \pm 0.0074 \text { and } r \leq 0.07 \text { with }\left|a_{\mathrm{S}}\right| \ll 0.01
$$

Here, BK14 data is taken by the BICEP2/Keck Array CMB polarization experiments up to and including the 2014 observing season. The bound on $r$ tightens to $r \leq 0.06$ [4], if we include BICEP2/Keck Array data from the 2015 observing season and combine them with 2015 Planck data. For a direct comparison of our findings with the obervational outputs in Ref. [2, 3], we also compute $r_{0.002}=16 \epsilon\left(\widehat{\phi}_{0.002}\right)$ where $\widehat{\phi}_{0.002}$ is the value of $\widehat{\phi}$ when the scale $k=0.002 / \mathrm{Mpc}$, which undergoes $N_{0.002}=N_{\star}+3.22$ e-foldings during $\mathrm{HI}$, crosses the horizon of $\mathrm{HI}-$ see Sec. 3.3 . 


\subsubsection{Theoretical Considerations}

We may qualify better our models by taking into account three additional restrictions of theoretical origin. In particular:

(a) Gauge-coupling Unification. One of the strongest motivation of our proposal is that $W$ in Eq. (1.1) leads not only to an inflationary era but also to the breaking of a GUT scale symmetry. In our introductory set-up the v.e.vs of $\bar{\Phi}$ and $\Phi$ break $U_{B-L}$ down to $\mathbb{Z}_{2}^{B-L}$. Indeed, minimizing $V_{\mathrm{HI}}$ in Eq. (2.16) we find that a SUSY vacuum arising after the end of HI with

$$
\langle S\rangle=0 \text { and }|\langle\Phi\rangle|=|\langle\bar{\Phi}\rangle|= \begin{cases}\sqrt{1-\sqrt{1-4 M^{2}}} \simeq M\left(1+M^{2} / 2\right) & \text { for MI, } \\ M & \text { for MII. }\end{cases}
$$

Note that other critical points of $V_{\mathrm{HI}}$ for MI are not accessible by our inflaton - cf. Ref. [22]. Although $\langle\Phi\rangle$ and $\langle\bar{\Phi}\rangle$ break spontaneously $U(1)_{B-L}$, no cosmic strings are produced at the SUSY vacuum, since this symmetry is already broken during HI. The contributions from the soft SUSY breaking terms can be safely neglected within contemporary SUSY, since the corresponding mass scale is much smaller than $M$. They may shift [16-18, 28], however, slightly $\langle S\rangle$ from zero in Eq. (3.6).

As regards the value of $M$, it can be determined by requiring that $\langle\Phi\rangle$ and $\langle\bar{\Phi}\rangle$ take the values dictated by the unification of MSSM gauge coupling constants. In particular, the unification scale $M_{\mathrm{GUT}} \simeq 2 / 2.433 \times 10^{-2} \simeq 8.22 \cdot 10^{-3}$ is to be identified with $M_{B L}-$ see Table $1-$ at the SUSY vacuum,

$$
\left\langle M_{B L}\right\rangle=2 \sqrt{N} g M /\left\langle f_{K}\right\rangle=M_{\mathrm{GUT}} \Rightarrow M \simeq M_{\mathrm{GUT}} / 2 g \sqrt{N} \text { for }\left\langle f_{K}\right\rangle \simeq 1 .
$$

Here $g \simeq 0.7$ is the value of the GUT gauge coupling constant. However, unification at a scale above $M_{\mathrm{GUT}}$ is also possible Ref. [51]. Also, $U(1)_{B-L}$ gauge symmetry does not disturb the MSSM unification. We can, therefore, treat $M$ as a free parameter keeping in mind that its most natural value is that given in Eq. (3.7) - see Sec. 3.3.

The determination of $M$ influences heavily the inflaton mass at the vacuum, $\widehat{m}_{\delta \phi}$ and induces an $N$ dependence in the results. Indeed, the (canonically normalized) inflaton,

$$
\widehat{\delta \phi}=\langle J\rangle \delta \phi \text { with } \delta \phi=\phi-M \text { and }\langle J\rangle=2 \sqrt{N /\left\langle f_{K}\right\rangle}
$$

acquires mass, at the SUSY vacuum in Eq. (3.6), which is given by

$$
\widehat{m}_{\delta \phi}=\left\langle V_{\mathrm{HI}, \widehat{\phi} \widehat{\phi}}\right\rangle^{1 / 2}=\left\langle V_{\mathrm{HI}, \phi \phi} / J^{2}\right\rangle^{1 / 2} \simeq \frac{\lambda M}{2 \sqrt{2 N}} \cdot \begin{cases}\sqrt{1-3 M^{2}} & \text { for MI, } \\ \left\langle f_{K}\right\rangle & \text { for MII, }\end{cases}
$$

where the last (approximate) equality for $\mathrm{MI}$ is valid only for $M \ll 1-$ see Eq. (3.6).

(b) Effective Field Theory. Destabilization of our inflationary scheme may be caused by higher order non-renormalizable terms in Eq. (1.1) and non-homogenous terms to the $K$ 's in Eq. (1.4) originated from non-perturbative (instanton) corrections within superstring theory - see Ref. [42]. To minimize the ramifications to our models from such terms, we impose two additional theoretical constraints - keeping in mind that $V_{\mathrm{HI}}\left(\phi_{\mathrm{f}}\right) \leq V_{\mathrm{HI}}\left(\phi_{\star}\right)$ :

$$
V_{\mathrm{HI}}\left(\phi_{\star}\right)^{1 / 4} \leq 1 \text { and } \phi_{\star} \leq 1
$$

The first from the inequalities above is easily satisfied in our set-up as inferred from Fig. 1. The second one is automatically fulfilled. Indeed, by construction $K$ 's in Eqs. (1.4) and (1.8) with the parameterizations in Eq. (2.6) induce a kinetic pole for $\phi_{\mathrm{p}}=1$ and so HI takes place for $\phi<1$. Even if we multiply all the quadratic terms in $K$ 's by a factor $c_{\Phi}$ - to keep the symmetries in Eq. 1.5 - with 
$c_{\Phi}<1$, then although $\phi_{\mathrm{p}}$ seems to be translated to transplanckian values, this is not the case. This is, because if we perform the rescalings

$$
\Phi \rightarrow \Phi / \sqrt{c_{\Phi}}, \bar{\Phi} \rightarrow \bar{\Phi} / \sqrt{c_{\Phi}} \text { and } S \rightarrow S,
$$

$c_{\Phi}$ can be absorbed in $K$ 's whereas $W$ of Eq. (1.1) remains unaltered provided we do the redefinitions

$$
\lambda \rightarrow c_{\Phi} \lambda, \quad \lambda^{\prime} \rightarrow c_{\Phi}^{2} \lambda^{\prime} \text { and } M \rightarrow M / \sqrt{c_{\Phi}} .
$$

As a consequence, we obtain a relocation of the pole at $\phi_{\mathrm{p}}=1$ and $\mathrm{HI}$ can be processed for subplanckian $\phi$ 's as analyzed in Sec. 3.2 below.

(c) Tuning of the Initial Conditions. HI may be implemented undoubtedly if $\phi$ starts its slow-roll below the location of kinetic pole. The closer to pole is set $\phi_{\star}$ the larger $N_{\star}$ is obtained. Therefore, a tuning of the initial conditions is required which can be somehow quantified - cf. Ref. [16-18, 20] defining the quantity

$$
\Delta_{\star}=\left(\phi_{\mathrm{p}}-\phi_{\star}\right) / \phi_{\mathrm{p}} .
$$

The naturalness of the attainment of $\mathrm{HI}$ increases with $\Delta_{\star}$.

\subsection{Analytic Results}

The investigation of the inflationary dynamics can be performed employing the formulae of Sec.3.1 and the expressions $V_{\mathrm{HI}}=V_{\mathrm{HI}}(\phi)$ in Eq. (2.16) taking advantage of $J$ in Eq. (2.13c) and making use of the chain rule of derivation - for more details see Ref. [19]. Following this strategy, we avoid to employ the quite complicate expression of $V_{\mathrm{HI}}=V_{\mathrm{HI}}(\widehat{\phi})$ in Eq. 2.17). We present our findings in Sec. 3.2.1 and 3.2.2 for MI and MII respectively.

\subsubsection{MODEL I (MI)}

Taking into account that no inflationary solutions are numerically localized for MI and $N \neq 1$ we confine ourselves to $N=1$ here and hereafter. The slow-roll parameters read

$$
\begin{aligned}
\epsilon & =2 \phi^{2}\left(\frac{1+\left(1+\delta_{\lambda}\right) \phi^{2}\left(\phi^{2}-2\right)-M^{2}}{\phi^{2}-\phi^{4}\left(1+\delta_{\lambda}\right) \phi^{4}-M^{2}}\right)^{2} ; \\
\eta & \simeq \frac{\left(3-5 \phi^{2}\right) f_{K}}{\phi^{2}}+\frac{\left(7 \phi^{2}-9\right) \delta_{\lambda} \phi^{4}+M^{2}\left(10 \phi^{4}-17 \phi^{2}+5\right)}{f_{K} \phi^{4}} .
\end{aligned}
$$

The condition of Eq. (3.2) in the present case reads

$$
\phi_{\mathrm{f}} \simeq \max (1 / \sqrt{2}, \sqrt{(9-\sqrt{21}) / 10}),
$$

i.e., $\phi_{\mathrm{f}}$ is determined due to the violation of the $\epsilon$ criterion. Assuming $\phi_{\mathrm{f}} \ll \phi_{\star}, N_{\star}$ can be approximately computed from Eq. (3.1) as follows

$$
N_{\star}=\int_{\phi_{\mathrm{f}}}^{\phi_{\star}} J^{2} \frac{V_{\mathrm{HI}}}{V_{\mathrm{HI}, \phi}} d \phi \simeq \frac{1}{2} \frac{\phi_{\star}^{2}}{f_{K \star}} \Rightarrow \phi_{\star} \simeq \sqrt{\frac{2 N_{\star}}{2 N_{\star}+1}} \text { where } f_{K \star}=1-\phi_{\star}^{2} .
$$

Also any dependence on the parameters $\delta_{\lambda}$ and $M$ can be safely neglected. Here we explicitly display the dependence of $N_{\star}$ on $\phi_{\star}$ to facilitate the understanding of the final result. It is clear that, as $\phi_{\star}$ approaches unity, $N_{\star}$ increases drastically assuring thereby the achievement of efficient HI. Indeed, from the last expression we infer that $\phi_{\star}$ is slightly lower than unity, as anticipated in Sec. 2.4. Plugging $\phi_{\star}$ from Eq. (3.16) into the rightmost equation in Eq. (3.1) and solving w.r.t $\lambda$, we arrive at the expression

$$
\lambda=8 \pi \sqrt{3 A_{\mathrm{s}}} \frac{1-4 M_{\lambda} N_{\star}^{2}}{N_{\star}\left(1-2 M_{\lambda} N_{\star}\right)^{2}} \text { with } M_{\lambda}=\delta_{\lambda}+M^{2} .
$$


Enforcing Eq. (3.3) on $A_{\mathrm{s}}$ we expect that $\lambda$ is comparable with its value in the quatric power-law model [2]. Upon substitution of $\phi_{\star}$ into Eq. (3.4) we obtain the the observational predictions of MI which are

$$
n_{\mathrm{s}} \simeq 1-2 M_{\lambda}\left(9+4 N_{\star}\right)-\frac{2}{N_{\star}}-\frac{48 N_{\star}^{2} M_{\lambda}^{2}}{\left(1-2 M_{\lambda} N_{\star}\right)^{2}}, r \simeq \frac{8\left(1-4 M_{\lambda} N_{\star}^{2}\right)^{2}}{N_{\star}^{2}\left(1-2 M_{\lambda} N_{\star}\right)^{2}} \text { and } a_{\mathrm{s}} \sim-\frac{3}{N_{\star}^{2}},
$$

where the last expression (for $a_{\mathrm{s}}$ ) yields just an order of magnitude estimation - the exact result is too lengthly to be worthily presented. Obviously, for $M_{\lambda}=0$, the well-known predictions of the $\mathrm{TM}_{4}$ in Eq. (1.3) are recovered, i.e., $n_{\mathrm{s}} \simeq 0.963, a_{\mathrm{s}} \simeq 6.3 \cdot 10^{-4}$ and $r \simeq 0.003$ for $N_{\star}=55$. As we can verify numerically, the expression above provide sufficiently accurate results within the whole allowed region of parameters presented in Sec. 3.3 .

\subsubsection{MODEL II (MII)}

Working along the lines of the previous section, we estimate the slow-roll parameters for MII as follows

$$
\epsilon=\frac{2 \phi^{2}}{N}\left(\frac{f_{K}}{\phi^{2}-M^{2}}\right)^{2} \text { and } \eta=\frac{f_{K}}{N} \frac{5 \phi^{4}-3\left(1+M^{2}\right) \phi^{2}+M^{2}}{\left(\phi^{2}-M^{2}\right)^{2}},
$$

where we keep now the $N$ dependence in the formulas. HI is over when Eq. (3.2) is saturated at the maximal $\phi$ value, $\phi_{\mathrm{f}}$, from the following two values

$$
\phi_{1 \mathrm{f}} \simeq \sqrt{4+N-\sqrt{N(N+8)}} / 2 \text { and } \phi_{2 \mathrm{f}} \simeq \sqrt{N+8-\sqrt{N^{2}+16 N+4}} / \sqrt{10}
$$

where $\phi_{1 \mathrm{f}}$ and $\phi_{2 \mathrm{f}}$ are such that $\epsilon\left(\phi_{1 \mathrm{f}}\right) \simeq 1$ and $\eta\left(\phi_{2 \mathrm{f}}\right) \simeq 1$. For $N>3$, the latter condition overshadows the former. Note that for $N=1$ the expressions above coincide with those in Eq. (3.15) since the slow-roll parameters for MI and MII tend to the same limit for $M=\delta_{\lambda}=0$ and $N=1$.

Assuming $\phi_{\star} \gg \phi_{\mathrm{f}}$ and neglecting subdominant logarithmic contributions, we can estimate $N_{\star}$ from Eq. (3.1) with result similar to that in Eq. 3.16, i.e.,

$$
N_{\star} \simeq \frac{N}{2} \frac{\phi_{\star}^{2}}{f_{K \star}}\left\langle f_{K}\right\rangle \Rightarrow \phi_{\star} \simeq \sqrt{\frac{N_{\star}}{\widetilde{N}_{\star}}} \text { with }\left\{\begin{array}{l}
\left\langle f_{K}\right\rangle=1-M^{2}, \\
\widetilde{N}_{\star}=N_{\star}+\left\langle f_{K}\right\rangle N / 2 .
\end{array}\right.
$$

Given that $\left\langle f_{K}\right\rangle \simeq 1$ and $N \ll N_{\star}$, we expect that $\phi_{\star}$ is again a little less than the pole value. Plugging $\phi_{\star}$ from Eq. (3.21) into the rightmost equation in Eq. (3.1) and solving w.r.t $\lambda$, we find

$$
\lambda \simeq 8 \sqrt{3 N A_{\mathrm{s}}} \pi\left\langle f_{K}\right\rangle / N_{\star}
$$

which results to similar numerical value with that found for MI. Inserting, finally, $\phi_{\star}$ from Eq. (3.21) into Eq. (3.4) and expanding successively the resulting equations in series of $M$ and $1 / N_{\star}$ we obtain

$$
n_{\mathrm{s}} \simeq 1-\frac{3\left\langle f_{K}\right\rangle}{N_{\star}}+\frac{\left\langle f_{K}\right\rangle}{\widetilde{N}_{\star}}, \quad r \simeq \frac{8 N\left\langle f_{K}\right\rangle^{2}}{N_{\star} \widetilde{N}_{\star}} \text { and } a_{\mathrm{s}} \simeq-\left(\frac{3}{N_{\star}^{2}}-\frac{1}{\widetilde{N}_{\star}}\right)\left\langle f_{K}\right\rangle^{2} .
$$

From the expressions above, we can infer that the predictions of pure $\mathrm{TM}_{4}$ in Eq. (1.3) are revealed for $N<10$ and $\left\langle f_{K}\right\rangle \simeq 1-$ recall that our results can be directly compared with those in Refs. [31,35,38] setting $2 N=3 \alpha$. Taking into account the definition of $\widetilde{N}_{\star}$ in Eq. (3.21) and solving the second formula above w.r.t $N$ we can also obtain the upper bound $N \lesssim 35$ imposing the second inequality in Eq. (3.5) for $N_{\star} \simeq 55-$ cf. Ref. [38]. This is in good agreement with our numerical findings, where $N_{\star}$ is consistently derived from Eq. (3.3). Numerically we also verify generically the correctness of the simple expressions above for $N>0.1$ and $M<0.3$. 


\subsection{Numerical Results}

Let us recall that our inflationary scenaria depends on the following parameters - see Eqs. (1.1), (1.4) and (1.8):

$$
M, \lambda \text { and } \delta_{\lambda} \text { for MI, or } N \text { for MII. }
$$

Note that $N_{S}$ in Eq. (1.2) does not affect the inflationary outputs, provided that $\widehat{m}_{s}^{2}>H_{\mathrm{HI}}^{2}$ for every allowed $N$. This is satisfied when $0<N_{S} \leq 6$, as explained in Sec. 2.4 Recall that we exclusively use $N=1$ for MI, since no acceptable inflationary solutions are found for other $N$ 's. This $N$ value can be regarded as a prediction than as a shortcoming of MI. The confrontation of the aforementioned parameters with observations is implemented as follows: Upon substitution of $V_{\mathrm{HI}}$ from Eq. (2.16) in Eqs. (3.1), (3.2) and (3.4) we extract the inflationary observables as functions of these parameters and $\phi_{\star}$. The last quantity and $\lambda$ can be determined by enforcing the fulfilment of Eq. (3.3), whereas $\delta_{\lambda}$ and $N$ largely affect the outputs on $n_{\mathrm{S}}$ and $r$ respectively and are constrained by Eq. (3.5). The predictions for $\widehat{m}_{\delta \phi}$ follow from Eq. (3.9).

We start the presentation of our results by comparing the outputs of our models against the observational data [2,3] in the $n_{\mathrm{s}}-r_{0.002}$ plane - see Fig. 2. More precisely, the dark [light] shaded contours in Fig. 2]depict the marginalized joint $68 \%$ [95\%] c.l. regions obtained by fitting the current data [2,3] with $\Lambda \mathrm{CDM}+r-$ see Fig. 28 of Ref. [1]. We draw dashed [solid] lines for MI [MII] and show the variation of $-\delta_{\lambda} / 10^{-5}[N]$ along each line fixing $\left\langle M_{B L}\right\rangle=M_{\mathrm{GUT}}$. Variation of $\left\langle M_{B L}\right\rangle-$ see below - leads to undistinguishable modifications of the displayed lines although the values of $\delta_{\lambda}$ and $N$ are to be readjusted. For most of the indicated points we list in the Table of Fig. 2 the corresponding field values $\left(\phi_{\star}\right.$ and $\left.\phi_{\mathrm{f}}\right)$, the output parameters $\left(\Delta_{\star}, \lambda\right.$ and $\left.M\right)$ and the inflationary observables. Note that, given $g\left(M_{\mathrm{GUT}}\right) \simeq 0.7$, the imposed constraint on $\left\langle M_{B L}\right\rangle$ determines uniquely $M$ for MI since $N=1$ whereas it allows for a $N$ dependence for MII - see Eq. (3.7). Recall that the variation of $V_{\mathrm{HI}}$ as a function of $\phi$ and $\widehat{\phi}$ is illustrated in Fig. 1. (a) and (b) respectively for MI and $\delta_{\lambda}=-4 \cdot 10^{-5}$ (light gray lines) or MII and $N=9$ (black lines).

Focusing first on MI, we see that the whole observationally favored range at low $r$ 's is covered varying $\left(-\delta_{\lambda}\right)$ which remains rather close to $10^{-5}$. In other words, $\mathrm{HI}$ is feasible only at the cost of a tuning of order $10^{-5}$ on $\delta_{\lambda}$ and another, of the order $10^{-2}$ on $\Delta_{\star}$. This amount of tuning is unavoidable, though, in this kind of models - cf. Ref. [9, 32,33]. The assumption $\delta_{\lambda}=0$ yields results identical to $\mathrm{TM}_{4}$ but it causes concerns regarding the naturality of the model since such arrangement requires the exact equality of the coefficients of two unrelated terms in $W$. In particular, let $n_{\mathrm{s}}$ vary in the range of Eq. (3.5) from the findings listed in the Table of Fig. 2, we obtain

$$
2.4 \lesssim \frac{-\delta_{\lambda}}{10^{-5}} \lesssim 5.2, \quad 4.6 \gtrsim \frac{\Delta_{\star}}{10^{-3}} \gtrsim 4.1, \quad 5.4 \lesssim \frac{-a_{\mathrm{S}}}{10^{-4}} \lesssim 8.6 \text { and } 2.1 \lesssim \frac{r}{10^{-3}} \lesssim 3.4 .
$$

Since MI predicts $r \gtrsim 0.0019$, it is testable by the forthcoming experiments, like BICEP3 [5], PRISM [6] and LiteBIRD [7], which are expected to measure $r$ with an accuracy of $10^{-3}$.

Turning now to MII, we observe from Fig. 2 that almost all the allowed $r$ 's are accessible by varying $N$ whereas $\Delta_{\star}$ increases with $N$. That is, the required tuning respecting the initial conditions becomes less and less severe as $N$ increases. We employ here just integer $N$ 's since these values are better motivated from the string theory. Comparing our present outputs with those of the models in Refs. [18,-20], where similar observable $r$ values are achieved with decimal $N$ values, we have to recognize that the quality of the $N$ adjustment is better here. On the other hand, $n_{\mathrm{s}}$ is concentrated a little lower than its central value in Eq. (3.5) without possibility of further variation without alteration to the required value of $N_{\star}$. From the results accumulated in the Table of Fig. 2 we find the following allowed ranges of parameters:

$$
0.962 \lesssim n_{\mathrm{s}} \lesssim 0.964, \quad 1 \lesssim N \lesssim 40, \quad 0.45 \gtrsim \Delta_{\star} / 10^{-2} \gtrsim 13.6 \text { and } 0.0025 \lesssim r \lesssim 0.07 .
$$




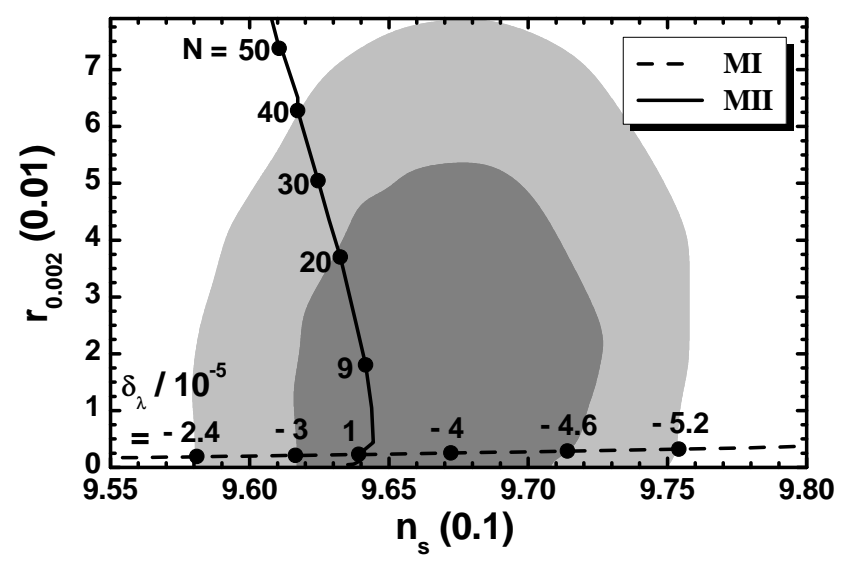

\begin{tabular}{|c||ccccc|ccccc|}
\hline Model: & \multicolumn{5}{c|}{ Model I (MI) } & \multicolumn{5}{c|}{ Model II (MII) } \\
\hline$\delta_{\lambda} / 10^{-5}$ & -2.4 & -3 & -4 & -4.6 & -5.2 & \multicolumn{5}{c}{-} \\
$N$ & & & 1 & & & 1 & 9 & 20 & 30 & 40 \\
\hline \hline$\phi_{\star} / 0.1$ & 9.9538 & 9.955 & 9.9564 & 9.958 & 9.9592 & 9.9554 & 9.6322 & 9.2402 & 8.9244 & 8.64 \\
$\Delta_{\star}(\%)$ & 0.46 & 0.45 & 0.44 & 0.42 & 0.41 & 0.45 & 3.7 & 7.6 & 10.7 & 13.6 \\
$\phi_{\mathrm{f}} / 0.1$ & & & 7.1 & & & 7.1 & 4.3 & 3.3 & 2.8 & 2.5 \\
\hline$N_{\star}$ & 55.6 & 55.7 & 55.1 & 55.7 & 55.8 & 55.2 & 56.8 & 57.2 & 57.4 & 57.5 \\
\hline$\lambda / 10^{-5}$ & 3.3 & 3.4 & 3.8 & 3.9 & 4.1 & 3.6 & 10.7 & 16.5 & 20.8 & 24.8 \\
$M / 10^{-3}$ & & & 5.87 & & & 5.87 & 1.96 & 1.31 & 1.075 & 0.93 \\
\hline$n_{\mathrm{s}} / 0.1$ & 9.58 & 9.62 & 9.67 & 9.71 & 9.75 & 9.64 & 9.64 & 9.63 & 9.62 & 9.62 \\
$-a_{\mathrm{s}} / 10^{-4}$ & 5.4 & 5.9 & 7.1 & 7.8 & 8.6 & 6.5 & 6.4 & 6.6 & 6.9 & 7 \\
$r / 10^{-2}$ & 0.21 & 0.23 & 0.28 & 0.31 & 0.34 & 0.25 & 2 & 4 & 5.5 & 6.9 \\
\hline
\end{tabular}

Figure 2: Allowed curves in the $n_{\mathrm{s}}-r_{0.002}$ plane fixing $M_{B L}=M_{\mathrm{GUT}}$ for $M I$ and various $\delta_{\lambda}$ 's indicated on the dashed line or MII and various $N$ 's indicated on the solid line. The marginalized joint $68 \%$ [95\%] c.l. regions from Planck TT, TE, EE+lowE+lensing, BK14 and BAO data [2] are depicted by the dark [light] shaded contours. The relevant field values, parameters and observables corresponding to points shown in the plot are listed in the Table.

Regarding $a_{\mathrm{s}}$, it varies in the range $-(6.3-7.1) \cdot 10^{-4}$ and so, MII is also consistent with the fitting of data with the $\Lambda \mathrm{CDM}+r$ model [2]. MII not only can be probed by the aforementioned future experiments but it can also become compatible with optimistic current central values of $r$ mentioned in Ref. [4]. E.g., we are able to achieve $r=0.02$ or $r=0.012$ with $N=9$ or $N=5.5$ correspondingly.

As we mention in Sec. 3.1, $\widehat{m}_{\delta \phi}$ is affected heavily from the choice of $M$. For the most natural $M$ choice done in Fig. 2, we obtain

$$
1.7 \lesssim \widehat{m}_{\delta \phi} / 10^{11} \mathrm{GeV} \lesssim 2.1 \text { or } 1.8 \lesssim \widehat{m}_{\delta \phi} / 10^{11} \mathrm{GeV} \lesssim 1.9
$$

for MI and MII respectively. Here and hereafter we restore units for convenience regarding the results on $\widehat{m}_{\delta \phi}$. These $\widehat{m}_{\delta \phi}$ ranges let, in principle, open the possibility of non-thermal leptogenesis if we introduce a suitable coupling between $\bar{\Phi}$ and the right-handed neutrinos - see e.g. Refs. [15-18]. This issue, though, needs further investigation in order to be surely verified.

Taking advantage from the fact that $M$ can be liberated from any gauge unification constraint see Sec. 3.1.2 - we investigate the modification of our results if we vary $M$ continuously from a low scale $M \simeq 0.001$ up to a maximal one which corresponds to $\left\langle M_{B L}\right\rangle=1$ with $g \simeq 0.7$. The indicative low bound on $M$ stems from the fact that $W$ in Eq. (1.1) is established around $m_{\mathrm{P}}$ and so we expect 


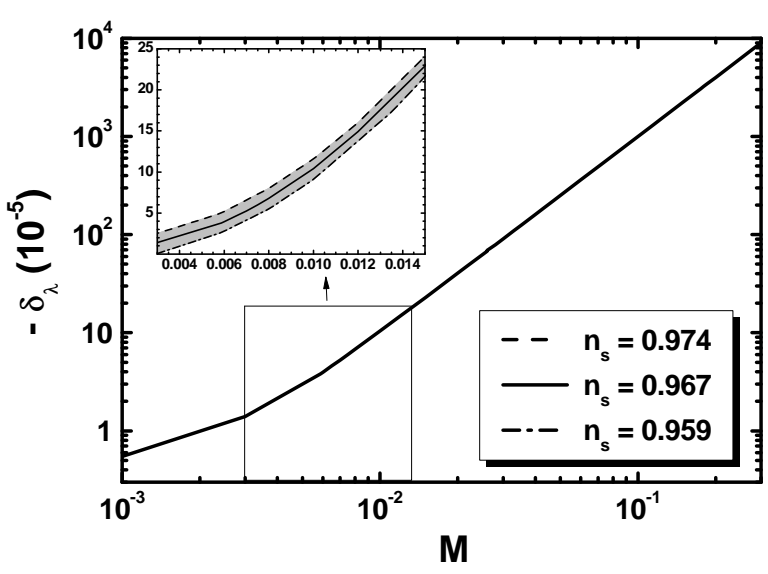

(a)

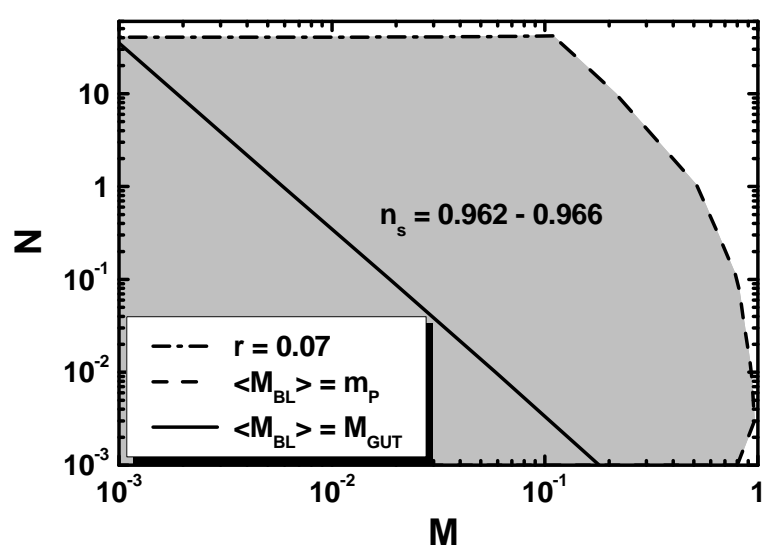

(b)

Figure 3: Allowed (shaded) regions in the (a) $M-\left(-\delta_{\lambda}\right)$ plane for $M I$ and (b) $M-N$ plane for MII. The conventions adopted for the various lines are also shown.

that the scale $M$, entered by hand in the theory, must have comparable size. Our outputs for MI and MII are presented in Fig. 3-(a) and (b) respectively and discussed below.

As regards MI, the allowed (shaded) region in the $M-\left(-\delta_{\lambda}\right)$ plane is bounded from the upper and lower limits on $n_{\mathrm{S}}$ in Eq. (3.5) which are depicted by a dashed and a dot-dashed line respectively - see the segment of the graph enclosed in a box and enlarged in the inset. These lines coincide with each other in the bulk of the plot. So we draw just a solid line corresponding to the central value of $n_{\mathrm{s}}$ in Eq. (3.5). Along this line in Fig. 3-(a) we get

$$
10^{-3} \lesssim M \lesssim 0.2, \quad 5.5 \cdot 10^{-4} \lesssim-\delta_{\lambda} / 10^{-2} \lesssim 4 \text { and } 3.2 \lesssim \widehat{m}_{\delta \phi} / 10^{10} \mathrm{GeV} \lesssim 644.7
$$

with $\lambda \sim 3.7 \cdot 10^{-5}, \Delta_{\star} \simeq 0.0043$ and $a_{\mathrm{s}} \simeq-0.00067$. For $M$ values beyond the upper bound above we do not succeed to achieve consistent inflationary solutions. We see that the tuning w.r.t $\delta_{\lambda}$ is ameliorated as $M$ increases, whereas that of $\Delta_{\star}$ remains unaltered.

On the other hand, the allowed (shaded) region for MII is delineated in the $M-N$ plane. Here we extend our scanning to non-integer $N$ too. We impose an artificial lower bound on $N$ stemming from naturalness. In fact, the tuning respecting $\Delta_{\star}$ is worsened as $N$ decreases and so enforcing $\Delta_{\star} \geq 10^{-6}$ applies $N \geq 10^{-3}$. The upper boundary curve of this region comes from the bound on $r$ in Eq. (3.5) which is depicted by a dot-dashed line and provides an (almost constant) upper bound on $N$. The rightmost border of the shaded region, represented by a dashed line, originates from the saturation of the expectation that $\left\langle M_{B L}\right\rangle \leq m_{\mathrm{P}}$. Along the solid line we obtain the theoretically better motivated value of $M$ corresponding to $\left\langle M_{B L}\right\rangle=M_{\mathrm{GUT}}$. Taking the logarithm of both sides of Eq. (3.7) with constant $g$, we can easily confirm the linear dependence of $\ln N$ on $\ln M$ with negative coefficient, in accordance with the depiction of the solid line in Fig. 3-(b). In the whole allowed region, we obtain

$$
\begin{gathered}
10^{-3} \lesssim M \lesssim 0.521, \quad 10^{-3} \lesssim N \lesssim 40, \quad 0.962 \lesssim n_{\mathrm{s}} \lesssim 0.966, \quad 2.9 \cdot 10^{-6} \lesssim r \lesssim 0.07 \\
5.3 \cdot 10^{-3} \lesssim \lambda / 10^{-4} \lesssim 3.7, \quad 10^{-6} \lesssim \Delta_{\star} \lesssim 0.136 \text { and } 0.3 \lesssim \widehat{m}_{\delta \phi} / 10^{11} \mathrm{GeV} \lesssim 721
\end{gathered}
$$

Meanwhile, $a_{\mathrm{S}}$ is confined in the range $-(6.1-7.4) \cdot 10^{-4}$. We remark a stronger variation of $\lambda, \Delta_{\star}$ and $\widehat{m}_{\delta \phi}$ here from that shown below Eq. (3.27) due to the variation of $N$ which drastically affects the parameters above - see Eqs. (3.21) and (3.22). Finally, since $\Delta_{\star}$ changes with $N$ its upper bound remains intact from the $M$ alteration and coincides with that in Eq. (3.25). 


\section{Conclusions}

We established, in the context of SUGRA, a new type of HI i.e., inflation employing as inflaton $\phi$ the radial components of a conjugate pair of Higgs fields. To be specific, in the current paper these Higgs fields implement the breaking of a gauge $U(1)_{B-L}$ symmetry at a scale $M$ which may assume a value compatible with MSSM unification. This is realized making use of a superpotential $W$ determined by an $R$ and the gauge symmetries and containing the first allowed non-renormalizable term - see Eq. (1.1). We judiciously selected two pairs of Kähler potentials, the "untilded" and the "tilded" ones - see Eqs. (1.4) and (1.8) -, which respect the symmetries above, parameterize hyperbolic spaces - see Eq. (1.5) - and give rise to two models of HI, MI and MII- see Eq. (1.9). Both models share the same function which relates the initial, $\phi$, with the canonically normalized inflaton, $\widehat{\phi}$ and includes a pole of order two - see Eq. (2.13c).

MI is relied on $W$ and the "untilded" $K$ 's which allow the pole to appear also in the SUGRA potential $V_{\mathrm{HI}}$. The denominator of $V_{\mathrm{HI}}$ though almost cancels out by constraining the scalar curvatures of the corresponding Kähler manifolds in Eq. (1.6) to the values $\mathcal{R}_{(11)^{2}}=-4$ or $\mathcal{R}_{21}=-3$ and the coefficients of the $W$ terms via the parameter $\delta_{\lambda}$ in Eq. (1.7). In sharp contrast, the pole remains invisible in $V_{\mathrm{HI}}$ of MII which is based on the more structured, "tilded" $K$ 's and employ only renormalizable terms in $W$. In this case, the scalar curvatures of the corresponding Kähler manifolds assume the values $\mathcal{R}_{(11)^{2}}=-4 / N$ or $\mathcal{R}_{21}=-3 / N$ with variable $N$.

Both models excellently match the observations by restricting the free parameters to reasonably ample regions of values. In particular, within MI any observationally acceptable $n_{\mathrm{s}}$ is attainable by tuning $\delta_{\lambda}$ to values of the order $10^{-5}$, whereas $r$ is kept at the level of $10^{-3}$ - see Eq. (3.24). On the other hand, MII avoids any tuning, larger $r$ 's are achievable as $N$ approaches its maximal allowed value 40 , while $n_{\mathrm{s}}$ lies close to its central value - see Eq. (3.25). The inflaton mass is collectively confined to the range $(1.7-2.1) \cdot 10^{11} \mathrm{GeV}$. Varying $M$ beyond its SUSY value, we obtain larger $\widehat{m}_{\delta \phi}$ 's and an mitigation of the tuning above within MI. In all cases, HI is realized with subplanckian values of $\phi$, stabilizing thereby our predictions from possible higher order terms in $W$, and can remain immune from the one-loop radiative corrections.

Finally, we would like to point out that, although we have restricted our discussion on the $G_{B-L}=$ $G_{\mathrm{SM}} \times U(1)_{B-L}$ gauge group, HI analyzed in this paper has in fact a general character and can be applied in many extensions of the MSSM. It can be realized within other GUTs, provided that $\bar{\Phi}$ and $\Phi$ consist a conjugate pair of Higgs superfields. If we adopt another GUT gauge group, the inflationary predictions are expected to be quite similar to the ones discussed here with possibly different analysis of the stability of the inflationary trajectory, since different Higgs superfield representations may be involved in implementing the $G_{B-L}$ breaking to $G_{\mathrm{SM}}$. Removing the scale $M$ from $W$ in Eq. (1.1) and abandoning the idea of grand unification, our HI can be realized even by the electroweak Higgs boson within next-to-MSSM - cf. Ref. [14]. In a such case the electroweak symmetry breaking can be processed taking into account the soft-SUSY breaking terms. Since our main aim here is just the establishment of the pole-induced HI, we opted to utilize the simplest possible GUT embedding. Within non-SUSY framework, MII can be also implemented within non-linear sigma models which share similar Kähler geometries. In such a case the potential, put by hand, is given by Eq. (2.16) swapping $\lambda^{2}$ with $\lambda$. As a consequence, the tuning on $\lambda$ is aggravated since we expect $\lambda \sim 10^{-8}$ see $\lambda$ values in the Table of Fig. 2. Moreover, in the absence of SUSY, the gauge hierarchy problem somehow addressed in SUSY frameworks - requires a special treatment.

ACKNOWLEDGMENTS I would like to thank G. Lazarides for useful discussions. This research work was supported by the Hellenic Foundation for Research and Innovation (H.F.R.I.) under the "First Call for H.F.R.I. Research Projects to support Faculty members and Researchers and the procurement of high-cost research equipment grant" (Project Number: 2251). 


\section{Appendix A: Mathematical Supplement}

We review here some mathematical properties regarding the geometrical structure of $\bar{\Phi}-\Phi$ moduli space - the geometry of the $S$ dependent part of the adopted $K$ 's is analyzed in Ref. [24]. We focus our attention on $K_{(11)^{2}}, \widetilde{K}_{(11)^{2}}$ in Sec. A.1 and $K_{21}, \widetilde{K}_{21}$ in Sec. A.2.

Let us, initially, recall that Riemannian metric, defined by the line element $d s_{K}^{2}$, and the scalar curvature, $\mathcal{R}_{K}$, associated with each of $K$ 's above are calculated employing the standard formulae [52]

$$
d s_{K}^{2}=K_{I \bar{J}} d Z^{I} d Z^{* \bar{J}} \text { and } \mathcal{R}_{K}=-K^{I \bar{J}} \partial_{I} \partial_{\bar{J}} \ln \left(\operatorname{det} M_{\bar{\Phi} \Phi}\right)
$$

where $M_{\bar{\Phi} \Phi}=\left(K_{I \bar{J}}\right)$ expresses the kinetic mixing in the inflationary sector. It is defined consistently with Eq. (2.8) but it is here computed without taking into account the constraint of Eq. (2.7).

\section{A.1 The Kähler Potentials $K_{(11)^{2}}$ AND $\widetilde{K}_{(11)^{2}}$}

Applying Eq. (A.1) for $K=K_{(11)^{2}}$ and $\widetilde{K}_{(11)^{2}}$ we find

$$
d s_{(11)^{2}}^{2}=\frac{2 N|d \Phi|^{2}}{\left(1-2|\Phi|^{2}\right)^{2}}+\frac{2 N|d \bar{\Phi}|^{2}}{\left(1-2|\bar{\Phi}|^{2}\right)^{2}} \text { and } \mathcal{R}_{(11)^{2}}=-\frac{4}{N}
$$

where $d s_{(11)^{2}}^{2}$ has a diagonal structure and is well defined imposing the restrictions $|\Phi|<1 / \sqrt{2}$ and $|\bar{\Phi}|<1 / \sqrt{2}$. Each of the two terms of $d s_{(11)^{2}}^{2}$ represents the extensively analyzed [36] Poincaré disc which is the non-compact two-dimensional manifold $S U(1,1) / U(1)$ with scalar curvature equal to one half of $\mathcal{R}_{(11)^{2}}$ [24]. It is well-known that $S U(1,1) / U(1)$ is also parameterized in the half-plane coordinates (usually notated by $T$ and $T^{*}$ ) which are related to the disc coordinates - e.g., $\Phi$ and $\Phi^{*}$ - through a Cayley transformation [35, 37, 38]. This parameterization, though, violates the gauge symmetry and so it is unappropriate for our purposes.

An element of the coset space $S U(1,1) / U(1)$ may be represented as [24,45]

$$
\mathcal{U}=\left(\begin{array}{cc}
\alpha & b \\
b^{*} & \alpha
\end{array}\right) \text { with } \alpha \in \mathbb{R}_{+}, b \in \mathbb{C} \text { and } \alpha^{2}-|b|=1
$$

Indeed, taking into account the definition of the coset space, we can convince ourselves that the matrix

$$
U=\mathcal{U P}=\left(\begin{array}{cc}
a & c \\
c^{*} & a^{*}
\end{array}\right) \text { with } \mathcal{P}=\operatorname{diag}\left(e^{i \vartheta}, e^{-i \vartheta}\right), \quad a=\alpha e^{i \vartheta} \text { and } c=b e^{i \vartheta},
$$

is an element of $S U(1,1)$ satisfying the definitive relations

$$
U^{\dagger} \eta_{11} U=\eta_{11} \text { and } \operatorname{det} U=1 \text { with } \eta_{11}=\operatorname{diag}(1,-1),
$$

since $|a|^{2}-|c|^{2}=1$. We can consider two elements $\mathcal{U}_{1} \in S U(1,1) / U(1)_{\Phi}$ and $\mathcal{U}_{2} \in S U(1,1) / U(1)_{\bar{\Phi}}$ acting on $\Phi$ and $\bar{\Phi}$ respectively according to the linear fractional transformations

$$
\sqrt{2} \Phi \rightarrow \frac{\alpha_{1} \sqrt{2} \Phi+b_{1}}{b_{1}^{*} \sqrt{2} \Phi+\alpha_{1}} \text { and } \sqrt{2} \bar{\Phi} \rightarrow \frac{\alpha_{2} \sqrt{2} \bar{\Phi}+b_{2}}{b_{2}^{*} \sqrt{2} \bar{\Phi}+\alpha_{2}}
$$

where $\alpha_{i}^{2}-\left|b_{i}\right|^{2}=1$ with $i=1,2$ and the elements $\alpha_{i}$ and $b_{i}$ are contained in $\mathcal{U}_{i}$. The transformations above do not violate the $U(1)_{B-L}$ symmetry, provided that the elements of $\mathcal{U}_{i}$ are charged under $U(1)_{B-L}$ as follows

$$
(B-L)\left(\alpha_{1}, b_{1}, \alpha_{2}, b_{2}\right)=(0,1,0,-1)
$$


It is straightforward to show that $d s_{(11)^{2}}^{2}$ in Eq. A.2) remains invariant under the transformations in Eq. (A.6) and so we conclude that $K_{(11)^{2}}$ and $\widetilde{K}_{(11)^{2}}$ parameterize $\mathcal{M}_{(11)^{2}}$.

It is well-known [52] that a SUGRA model is fully described by the function $G$, see Eq. (2.2a), which remains invariant under a Kähler transformation

$$
K \rightarrow K+\Lambda+\Lambda^{*} \text { and } W \rightarrow W e^{-\Lambda}
$$

Therefore, models described by $K$ 's and $W$ 's related by such a transformation are equivalent. We can prove that $K_{(11)^{2}}$ in Eq. (1.4) remains invariant, in the sense of Eq. (A.8), i.e., it is transformed as in Eq. (A.8) with

$$
\Lambda=N \ln \left(\left(b_{1}^{*} \sqrt{2} \Phi+\alpha_{1}\right)\left(b_{2}^{*} \sqrt{2} \bar{\Phi}+\alpha_{2}\right)\right) .
$$

Similar transformation is valid for $\widetilde{K}_{(11)^{2}}$ in Eq. (1.8) since it is related to $K_{(11)^{2}}$ via another Kähler transformation.

\section{A.2 The Kähler Potentials $K_{21}$ ANd $\widetilde{K}_{21}$}

For $K=K_{21}$ and $\widetilde{K}_{21}$, Eq. (A.1) yields

$$
d s_{21}^{2}=2 N\left(\frac{|d \Phi|^{2}+|d \bar{\Phi}|^{2}}{1-|\Phi|^{2}-|\Phi|^{2}}+\frac{\left|\Phi^{*} d \Phi+\bar{\Phi}^{*} d \bar{\Phi}\right|^{2}}{\left(1-|\Phi|^{2}-|\bar{\Phi}|^{2}\right)^{2}}\right) \quad \text { and } \quad \mathcal{R}_{21}=-\frac{3}{N}
$$

where $d s_{21}^{2}$ is known in the mathematical literature - see e.g. Ref. [53] - as the Bergmann metric defined in the open ball $|\Phi|^{2}+|\bar{\Phi}|^{2}<1$ of $\mathbb{C}^{2}$. This is the non-compact, four-dimensional manifold $\mathcal{M}_{21}$ in Eq. (1.5). The value of $\mathcal{R}_{21}$ above is in accord with the generic result found in Ref. [34], if we take into account the different sign adopted in the definition of $\mathcal{R}_{K}$ in Eq. A.1 - recall that the transition between the two notations is realized setting $2 N=3 \alpha$. As for the case of $\mathcal{M}_{(11)^{2}}$, alternative parameterizations of $\mathcal{M}_{21}$ [33, 45, 48] are not allowed in our case, since these are not consistent with the gauge symmetry.

We can find a representative element of $\mathcal{M}_{21}$ following the method presented in Ref. [54]. Namely, an element of $S U(2,1)$ satisfying the definitive relations

$$
U^{\dagger} \eta_{21} U=\eta_{21} \text { and } \operatorname{det} U=1 \text { with } \eta_{21}=\operatorname{diag}(1,1,-1) \text {, }
$$

may be written as

$$
U=\mathcal{U P} \text { with } \mathcal{U}=\left(\begin{array}{ccc}
1 / N_{a} & 0 & a \\
N_{a} b a^{*} & N_{a} \gamma & b \\
N_{a} \gamma a^{*} & N_{a} b^{*} & \gamma
\end{array}\right) \quad \text { and } \mathcal{P}=e^{i \vartheta}\left(\begin{array}{ccc}
d & f & 0 \\
-f^{*} & d^{*} & 0 \\
0 & 0 & e^{-3 i \vartheta}
\end{array}\right)
$$

where $N_{a}=1 / \sqrt{1+|a|^{2}}$ and the free parameters $a, b, \gamma, d$ and $f$ are constrained as follows

$$
a, b, d, f \in \mathbb{C}, \gamma \in \mathbb{R}_{+} \text {with }|a|^{2}+|b|^{2}-\gamma^{2}=-1 \text { and }|d|^{2}+|f|^{2}=1 .
$$

The former constraint ensures the hyperbolic structure of $\mathcal{M}_{21}$ whereas the latter indicates the compact form of $S U(2)-$ cf. Ref. [24]. Obviously, $\mathcal{P} \in S U(2) \times U(1)-$ which is subgroup of $S U(2,1)-$ and depends on four (real) parameters. Consequently, $\mathcal{U}$ is a representative of $\mathcal{M}_{21}$ depending on four parameters. It is trivial to verify that the constraints in Eq. A.11) are fulfilled for $U=\mathcal{U}$ and $U=\mathcal{P}$ and so for $U=\mathcal{U P}$ too. As a cross check, recall that the pseudo-unitary group $S U(2,1)$ depends on eight (4+4) free parameters - cf. Ref. [45]. 
The operation of $\mathcal{U} \in \mathcal{M}_{21}$ on $\Phi$ and $\bar{\Phi}$ can be represented via the isometric transformations - cf. Eq. A.6

$$
\Phi \rightarrow \frac{\left(1 / N_{a}\right) \Phi+N_{a} b^{*} a \bar{\Phi}+N_{a} a \gamma}{a^{*} \Phi+b^{*} \bar{\Phi}+\gamma} \text { and } \bar{\Phi} \rightarrow \frac{N_{a} \gamma \bar{\Phi}+N_{a} b}{a^{*} \Phi+b^{*} \bar{\Phi}+\gamma},
$$

where the $\Phi$ and $\bar{\Phi}$ independent parameters originate from the lines of $\mathcal{U}^{\dagger}$. To keep consistency with the $U(1)_{B-L}$ symmetry we assign to the free parameters of $\mathcal{U}$ the $B-L$ charges

$$
(B-L)(a, b, \gamma)=(1,-1,0) .
$$

It is straightforward to show that $d s_{21}^{2}$ in Eq. A.10) remains invariant under Eq. A.14) and so, we conclude that $K_{21}$ and $\widetilde{K}_{21}$ parameterize $\mathcal{M}_{21}$. Moreover, $K_{21}$ in Eq. (1.4) and $\widetilde{K}_{21}$ in Eq. (1.8) remain invariant under Eq. (A.14), up to a Kähler transformation. E.g., $K_{21}$ is transformed as $K$ in Eq. (A.8) with

$$
\Lambda=2 N \ln \left(a^{*} \Phi+b^{*} \bar{\Phi}+\gamma\right) .
$$

To our knowledge, the representation of $\mathcal{U}$ in Eq. (A.12) and the isometries in Eq. (A.14) are employed for the first time in demonstrating the invariance of $d s_{21}^{2}$ and $K_{21}$ under $S U(2,1) /(S U(2) \times U(1))$.

\section{REFERENCES}

[1] N. Aghanim et al. [Planck Collaboration], Planck 2018 results. VI. Cosmological parameters Astron. Astrophys. 641, A6 (2020) [arXiv: 1807.06209 ].

[2] Y. Akrami et al. [Planck Collaboration], Planck 2018 results. X. Constraints on inflation, Astron. Astrophys. 641, A10 (2020) [arXiv: 1807.06211].

[3] P.A.R. Ade et al. [BICEP2/Keck Array Collaborations], Improved Constraints on Cosmology and Foregrounds from BICEP 2 and Keck Array Cosmic Microwave Background Data with Inclusion of 95 GHz Band, Phys. Rev. Lett. 116, 031302 (2016) [arXiv: 1510 .09217].

[4] P.A.R. Ade et al. [BICEP2/Keck Array Collaborations], BICEP2 / Keck Array x: Constraints on Primordial Gravitational Waves using Planck, WMAP, and New BICEP2/Keck Observations through the 2015 Season, Phys. Rev. Lett. 121, 221301 (2018) [arXiv:1810.05216].

[5] W.L.K. Wu et al., Initial Performance of BICEP3: A Degree Angular Scale 95 GHz Band Polarimeter, J. Low. Temp. Phys. 184, no. 3-4, 765 (2016) [arXiv: 1601.00125 ].

[6] P. Andre et al. [PRISM Collaboration], PRISM (Polarized Radiation Imaging and Spectroscopy Mission): A White Paper on the Ultimate Polarimetric Spectro-Imaging of the Microwave and Far-Infrared Sky, arXiv:1306.2259.

[7] L. Montier et al. [LiteBIRD], Overview of the Medium and High Frequency Telescopes of the LiteBIRD satellite mission, Proc. SPIE Int. Soc. Opt. Eng. 11443, 114432G (2020) [arXiv : 2102 . 00809 ].

[8] M. Yamaguchi, Supergravity based inflation models: a review, Class. Quant. Grav. 28, 103001 (2011) [arXiv:1101.2488]; J. Martin, C. Ringeval and V. Vennin, Encyclopadia Inflationaris, Phys. Dark Univ. 5, 75 (2014) [arXiv:1303.3787]; J. Martin, C. Ringeval R. Trotta and V. Vennin, The Best Inflationary Models After Planck, J. Cosmol. Astropart. Phys. 03, 039 (2014)14 [arXiv:1312.3529]; K. Sato and J. Yokoyama, Inflationary cosmology: First 30+ years, Int. J. Mod. Phys. D 24, no. 11, 1530025 (2015).

[9] J. Ellis, M.A.G. Garcia, N. Nagata, D.V. Nanopoulos, K.A. Olive and S. Verner, Building Models of Inflation in NoScale Supergravity, Int. J. Mod. Phys. D 29, 16, 2030011 (2020) [arXiv: 2009 . 01709].

[10] D.S. Salopek, J.R. Bond and J.M. Bardeen, Designing Density Fluctuation Spectra in Inflation, Phys. Rev. D 40, 1753 (1989); J.L. Cervantes-Cota and H. Dehnen, Induced gravity inflation in the SU(5) GUT, Phys. Rev. D 51, 395 (1995) [astro-ph/9412032].

[11] J.L. Cervantes-Cota and H. Dehnen, Induced gravity inflation in the standard model of particle physics, Nucl. Phys. B442, 391 (1995) [astro-ph/9505069]; F.L. Bezrukov and M. Shaposhnikov, Standard Model Higgs boson mass from inflation, Phys. Lett. B 659, 703 (2008) [arXiv: 0710 . 3755]. 
[12] N. Kaloper, L. Sorbo and J. Yokoyama, Inflation at the GUT scale in a Higgsless universe, Phys. Rev. D 78, 043527 (2008) [arXiv:0803.3809].

[13] S. Antusch et al., Gauge Non-Singlet Inflation in SUSY GUTs J. High Energy Phys. 08, 100 (2010) [arXiv: 1003.3233 ]; M. Arai, S. Kawai, and N. Okada, Higgs inflation in minimal supersymmetric SU(5) GUT, Phys. Rev. D 84,1 23515 (2011) [arXiv:1107. 4767]; K. Nakayama and F. Takahashi, PeV-scale Supersymmetry from New Inflation, J. Cosmol. Astropart. Phys. 05, 035 (2012) [arXiv:1203.0323]; M.B. Einhorn and D.R.T. Jones, GUT Scalar Potentials for Higgs Inflation, J. Cosmol. Astropart. Phys. 11, 049 (2012) [arXiv: 1207.1710 ]; M. Arai, S. Kawai, and N. Okada, Supersymmetric B-L inflation near the conformal coupling, Phys. Lett. B 734, 100 (2014) [arXiv:1311.1317]; I. Garg and S. Mohanty, No scale SUGRA SO(10) derived Starobinsky Model of Inflation, Phys. Lett. B 751, 7 (2015) [arXiv: 1504 .07725].

[14] M.B. Einhorn and D.R.T. Jones, Inflation with Non-minimal Gravitational Couplings in Supergravity, J. High Energy Phys. 03, 026 (2010) [arXiv:0912.2718]; S. Ferrara et al., Superconformal Symmetry, NMSSM, and Inflation, Phys. Rev. D 83, 025008 (2011) [arXiv: 1008 . 2942]; I. Ben-Dayan and M.B. Einhorn, Supergravity Higgs Inflation and Shift Symmetry in Electroweak Theory J. Cosmol. Astropart. Phys. 12, 002 (2010) [arXiv: 1009.2276 ]; K. Nakayama and F. Takahashi, Higgs Chaotic Inflation in Standard Model and NMSSM, J. Cosmol. Astropart. Phys. 02, 010 (2011) [arXiv: 1008.4457 ]; G. K. Chakravarty et al., Plateau Inflation in SUGRA-MSSM, Phys. Lett. B 760, 263 (2016) [arXiv: 1604 . 02556]; J. Ellis, H.J. He and Z.Z. Xianyu, New Higgs Inflation in a No-Scale Supersymmetric SU(5) GUT, Phys. Rev. D 91, no. 2, 021302 (2015) [arXiv: 1411.5537 ]; L.E. Ibáñez, F. Marchesano and I. Valenzuela, Higgs-otic Inflation and String Theory J. High Energy Phys. 01, 128 (2015) [arXiv: 1411.5380 ].

[15] C. Pallis and N. Toumbas, Non-Minimal Higgs Inflation and non-Thermal Leptogenesis in a Supersymmetric PatiSalam Model, J. Cosmol. Astropart. Phys. 12, 002 (2011) [arXiv:1108.1771]; C. Pallis and N. Toumbas, Leptogenesis and Neutrino Masses in an Inflationary SUSY Pati-Salam Model, Open Questions in Cosmology (InTech, 2012) [arXiv:1207.3730].

[16] C. Pallis, Unitarity-Safe Models of Non-Minimal Inflation in Supergravity, Eur. Phys. J. C 78, no.12, 1014 (2018) [arXiv: 1807.01154 ]; C. Pallis, Unitarizing non-Minimal Inflation via a Linear Contribution to the Frame Function, Phys. Lett. B 789, 243 (2019) [arXiv: 1809.10667 ].

[17] C. Pallis and Q. Shafi, Induced-Gravity GUT-Scale Higgs Inflation in Supergravity, Eur. Phys. J. C 78, no.6, 523 (2018) [arXiv:1803.00349].

[18] C. Pallis, Gravitational Waves, $\mu$ Term \& Leptogenesis from B - L Higgs Inflation in Supergravity, Universe 4, no.1, 13 (2018) [arXiv: 1710 . 05759]; C. Pallis, B - L Higgs Inflation in Supergravity With Several Consequences, PoS CORFU2017 (2018), 086 [arXiv: 1804 .07038].

[19] G. Lazarides and C. Pallis, Shift Symmetry and Higgs Inflation in Supergravity with Observable Gravitational Waves, J. High Energy Phys. 11, 114 (2015) [arXiv: 1508 .06682].

[20] C. Pallis, Kinetically Modified Non-Minimal Higgs Inflation in Supergravity, Phys. Rev. D 92, no. 12, 121305(R) (2015) [arXiv: 1511.01456]; C. Pallis, Variants of Kinetically Modified Non-Minimal Higgs Inflation in Supergravity, J. Cosmol. Astropart. Phys. 10, no. 10, 037 (2016) [arXiv: 1606.09607 ]; C. Pallis, Observable Gravitational Waves From Higgs Inflation in SUGRA, PoS EPS-HEP2017, 047 (2017) [arXiv: 1710.04641 ].

[21] G.R. Dvali, Q. Shafi and R.K. Schaefer, Large scale structure and supersymmetric inflation without fine tuning, Phys. Rev. Lett. 73, 1886 (1994) |hep-ph/9406319|.

[22] R. Jeannerot, S. Khalil, G. Lazarides and Q. Shafi, Inflation and Monopoles in Supersymmetric $S U(4)_{\mathrm{c}} \times S U(2)_{L} \times$ $S U(2)_{R}$, J. High Energy Phys. 10, 012 (2000) [hep-ph/ 0002151$]$.

[23] R. Kallosh, A. Linde and T. Rube, General inflaton potentials in supergravity, Phys. Rev. D 83, 043507 (2011) [arXiv:1011.5945].

[24] C. Pallis and N. Toumbas, Starobinsky-Type Inflation With Products of Kähler Manifolds, J. Cosmol. Astropart. Phys. 05, no. 05, 015 (2016) [arXiv: 1512.05657].

[25] H.M. Lee, Chaotic inflation in Jordan frame supergravity, J. Cosmol. Astropart. Phys. 08, 003 (2010) [arXiv:1005.2735].

[26] I. Antoniadis, E. Dudas, S. Ferrara and A. Sagnotti, The Volkov-Akulov-Starobinsky supergravity, Phys. Lett. B 733, 32 (2014) [arXiv: 1403 . 3269]; S. Ferrara, R. Kallosh and A. Linde, Cosmology with Nilpotent Superfields, J. High Energy Phys. 10, 2014 (143) [arXiv: 1408 .4096]. 
[27] S.V. Ketov and T. Terada, Inflation in supergravity with a single chiral superfield, Phys. Lett. B 736, 272 (2014) [arXiv:1406.0252].

[28] C. Pallis, Linking Starobinsky-Type Inflation in no-Scale Supergravity to MSSM, J. Cosmol. Astropart. Phys. 04, 024 (2014); Erratum-ibid. 07, 01(E) (2017) [arXiv: 1312 . 3623].

[29] C. Pallis, Induced-Gravity Inflation in no-Scale Supergravity and Beyond, J. Cosmol. Astropart. Phys. 08, 057 (2014) [arXiv:1403.5486]; C. Pallis, Reconciling Induced-Gravity Inflation in Supergravity with the Planck 2013 \& BICEP2 Results, J. Cosmol. Astropart. Phys. 10, 058 (2014) [arXiv: 1407 . 8522].

[30] G.F. Giudice and H.M. Lee, Starobinsky-like inflation from induced gravity, Phys. Lett. B 733, 58 (2014) [arXiv:1402.2129]; R. Kallosh, More on Universal Superconformal Attractors, Phys. Rev. D 89, no. 8, 087703 (2014) [arXiv: 1402.3286$]$.

[31] R. Kallosh and A. Linde, Universality Class in Conformal Inflation, J. Cosmol. Astropart. Phys. 07, 002 (2013) [arXiv:1306.5220].

[32] J. Ellis, D.V. Nanopoulos and K.A. Olive, No-Scale Supergravity Realization of the Starobinsky Model of Inflation, Phys. Rev. Lett. 111, 111301 (2013); Erratum-ibid. 111, no. 12, 129902 (2013) [arXiv: 1305.1247 ].

[33] J. Ellis, D. Nanopoulos and K. Olive, Starobinsky-like Inflationary Models as Avatars of No-Scale Supergravity, J. Cosmol. Astropart. Phys. 10, 009 (2013) [arXiv: 1307 . 3537].

[34] J. Ellis, D.V. Nanopoulos, K.A. Olive and S. Verner, Unified No-Scale Attractors, J. Cosmol. Astropart. Phys. 09, 2019 (040) [arXiv:1906.10176].

[35] R. Kallosh, A. Linde, and D. Roest, Superconformal Inflationary a-Attractors, J. High Energy Phys. 11, 198 (2013) [arXiv:1311.0472].

[36] R. Kallosh and A. Linde, Escher in the Sky, Comptes Rendus Physique 16, 914 (2015) [arXiv: 1503.06785$].$

[37] J.J.M. Carrasco, R. Kallosh, A. Linde and D. Roest, Hyperbolic geometry of cosmological attractors, Phys. Rev. D 92, no. 4, 041301 (2015) [arXiv: 1504 .05557]; J.J.M. Carrasco, R. Kallosh and A. Linde, Cosmological Attractors and Initial Conditions for Inflation, Phys. Rev. D 92, no.6, 063519 (2015) [arXiv: 1506.00936 ].

[38] S. Ferrara and R. Kallosh, Seven-disk manifold, $\alpha$-attractors, and B modes, Phys. Rev. D 94, no.12, 126015 (2016) [arXiv:1610.04163].

[39] M. Galante, R. Kallosh, A. Linde, and D. Roest, Unity of Cosmological Inflation Attractors, Phys. Rev. Lett. 114, no. 14, 141302 (2015) [arXiv: 1412.3797].

[40] S.V. Ketov, On the equivalence of Starobinsky and Higgs inflationary models in gravity and supergravity, J. Phys. A 53, no.8, 084001 (2020) [arXiv: 1911.01008].

[41] T. Terada, Generalized Pole Inflation: Hilltop, Natural, and Chaotic Inflationary Attractors, Phys. Lett. B 760, 674 (2016) [arXiv:1602.07867].

[42] B.J. Broy, M. Galante, D. Roest and A. Westphal, Pole inflation, Shift symmetry and universal corrections, J. High Energy Phys. 12, 149 (2015) [arXiv: 1507 .02277].

[43] T. Kobayashi, O. Seto and T.H. Tatsuishi, Toward pole inflation and attractors in supergravity : Chiral matter field inflation, Prog. Theor. Phys. 123B04, no.12 (2017) [arXiv: 1703.09960$]$; S. Karamitsos, Beyond the Poles in Attractor Models of Inflation, J. Cosmol. Astropart. Phys. 09, 022 (2019) [arXiv: 1903 . 0370 7]; J. Fumagalli, Renormalization Group independence of Cosmological Attractors, Phys. Lett. B 769, 451 (2017) [arXiv: 1611.04997 ].

[44] J. Ellis, D.V. Nanopoulos, K.A. Olive and S. Verner, Phenomenology and Cosmology of No-Scale Attractor Models of Inflation, J. Cosmol. Astropart. Phys. 08, 037 (2020) [arXiv: 2004 . 0064 3].

[45] J. Ellis, D.V. Nanopoulos, K.A. Olive and S. Verner, A general classification of Starobinsky-like inflationary avatars of $S U(2,1) / S U(2) \times U(1)$ no-scale supergravity, J. High Energy Phys. 03, 099 (2019) [arXiv : 1812 . 02192].

[46] K. Nakayama, K. Saikawa, T. Terada and M. Yamaguchi, Structure of Kähler potential for D-term inflationary attractor models, J. High Energy Phys. 05, 067 (2016) [arXiv: 1603.02557$].$

[47] A. Brignole, F. Feruglio and F. Zwirner, Higgs and super Higgs effects with naturally vanishing vacuum energy, Phys. Lett. B 356, 500 (1995) [hep-th/ 9504032 ]. 
[48] F. Farakos, A. Kehagias and A. Riotto, On the Starobinsky Model of Inflation from Supergravity, Nucl. Phys. B876, 187 (2013) [arXiv:1307.1137].

[49] Y. Ema et al., Supersymmetric Flaxion, J. High Energy Phys. 04, 094 (2018) [arXiv: 1802.07739 ].

[50] A. Kehagias and A. Riotto, Remarks about the Tensor Mode Detection by the BICEP2 Collaboration and the SuperPlanckian Excursions of the Inflaton Field, Phys. Rev. D 89, 101301 (2014) [arXiv: 1403.4811 ].

[51] C. Bachas, C. Fabre and T. Yanagida, Natural gauge coupling unification at the string scale, Phys. Lett. B 370, 49 (1996) [hep-th/9510094]; R. Howl and S.F. King, Planck scale unification in a supersymmetric standard model, Phys. Lett. B 652, 331 (2007) [arXiv:0705.0301].

[52] See, e.g., P. Binétruy, Supersymmetry: Theory, experiment and cosmology, Oxford (2006).

[53] M. Kapovich, Lectures On Complex Hyperbolic Kleinian Groups, arXiv:1911.12806 [math.GR].

[54] G. Khanna, S. Mukhopadhyay, R. Simon and N. Mukunda, Geometric Phases for SU(3) Representations and Three Level Quantum Systems, Ann. Phys. (NY) 253, 55 (1997). 